\title{
Asymptotic behavior and inverse problem in layered scattering media.
}

Jean-Michel Tualle, Ha Lien Nghiem, Dominique Ettori, Raphael Sablong, Eric Tinet and Sigrid Avrillier

Laboratoire de Physique des Lasers (CNRS UMR 7538), Université Paris 13

99 av. J.-B. Clément, 93430 Villetaneuse, France

\section{ABSTRACT:}

The main challenge of non invasive optical biopsy is to obtain an accurate value of the optical coefficients of an encapsulated organ (muscle, brain, ...). The idea developed here is that some interesting information could be deduced from the long time behavior of the reflectance function. This asymptotic behavior is analyzed for layered media, in the framework of the diffusion approximation. A new method is derived in order to obtain accurate values for the optical parameters of the deepest layers. This method is designed to work in a specific "long time" regime which is still in the scope of standard time of flight experiments, but far from being included into the mathematically defined asymptotic region. The limits of this method, linked to the cases where the asymptotic behavior is no more governed by the deepest layer, are then discussed.

( OCIS : 030-5620, 110-7050, 100-3190) 


\section{1)- Introduction.}

The real challenge of non invasive optical biopsy is to obtain an accurate in-vivo evaluation of the optical coefficients of an embedded organ from reflectance measurements. There was recently a lot of activities on light propagation and inverse problems in two-layered scattering media ${ }^{[1-6]}$. It appears from these studies that it is very difficult to obtain an accuracy better than $5 \%$ for the optical coefficients of the bottom layer ${ }^{[2,4]}$. Moreover, the performances of such an inversion should considerably be decreased when considering more than two layers. An interesting way to overcome these difficulties was recently proposed by Pifferi et al ${ }^{[7]}$ who used the knowledge of the absorption spectra of the main absorbers present in the medium to reconstruct the absorption coefficient. Their work concerns two-layered media and very accurate results were obtained even with a very thick $(1.2 \mathrm{~cm})$ first layer. Their method, which gives encouraging results, was however tested with a limited number of very contrasted dies, and cannot give any value for the reduced scattering coefficients.

It is a very common assertion to say that another way to obtain the optical properties of an organ covered by layers of tissues is to select the light that stays a "long time" in the medium and therefore spends most time in the deepest layer: One would then obtain results that more specifically depend on the deepest layer optical properties, and not as much on the properties of the overlying layers. In fact one main problem in that kind of approaches is that the times which are experimentally considered as long, are still far from the mathematically defined asymptotic region.

This paper is devoted to the analysis of this problem in the framework of the diffusion approximation. The long time behavior of the reflectance function in scattering media with $\mathrm{N}$ $\geq 2$ layers is explored and a method is derived to obtain the optical coefficients of the deepest layer at realistic "long times". This method is illustrated with different two-layered media. 


\section{2)- Reflectance function in scattering media with $\mathrm{N} \geq 2$ layers.}

Let us recall the well-known formulation of the diffusion approximation ${ }^{[8-13]}$ in layered media: In the medium illustrated in figure 1, each layer $i$ is characterized by an absorption coefficient $\mu_{a_{i}}$, a reduced scattering coefficient $\mu_{s i}^{\prime}$ and a diffusion constant $D_{i} \sim 1 / 3 \mu_{s i}^{\prime}$. The $\mathrm{z}$ axis is oriented towards the deepest layer, and the interface between layer $i$ and layer $i+1$ is located at $z=l_{i}$. The incident laser pulse is introduced through a point-like source located at $z=z_{0}{ }^{[8]}$. The diffuse photon fluence rate $\varphi$ satisfies the diffusion equation in each layer:

$$
\frac{1}{c_{i}} \frac{\partial \varphi}{\partial t}-D_{i} \Delta \varphi+\mu_{a i} \varphi=S
$$

Where $c_{i} \approx c_{0} / n_{i}$ is the light speed in layer $i$ and where the source term can be written $S(\vec{\rho}, z, t)=\delta(\vec{\rho}) \delta(t) \delta\left(z-z_{0}\right)$ for one incident unit of energy; $\vec{\rho}$ denotes the transverse plan coordinates.

Concerning the boundary conditions between two layers, one can write at $z=l_{i}{ }^{[1,10]}$ :

$$
\begin{aligned}
& n_{i+1}^{2} \varphi\left(l_{i}^{-}\right)=n_{i}^{2} \varphi\left(l_{i}^{+}\right) \\
& D_{i} \frac{\partial \varphi}{\partial z}\left(l_{i}^{-}\right)=D_{i+1} \frac{\partial \varphi}{\partial z}\left(l_{i}^{+}\right)
\end{aligned}
$$

For the free surface boundary condition, we can use the Extrapolated Boundary Condition $^{[11-13]}$ :

$$
\varphi\left(-z_{b}\right)=0
$$

where $z_{b}$ is an extrapolated distance which takes into account the index mismatch between air and medium 1 . For instance we will consider in the following the case $n_{l}=1.4$, which leads to $z_{b}=1.9 / \mu_{s 1}^{\prime}$. The last condition is of course $\varphi \rightarrow 0$ when $z \rightarrow \infty$.

This problem can be solved by using the Fourier Transform $\widetilde{\varphi}$ in time and transverse plane coordinates, which is a function of the conjugate variables of $t$ and $\vec{\rho}$ that are $\omega$ and 
$\vec{k}$ respectively. Kienle et al ${ }^{[1]}$ gave a solution in the case of a two-layered medium. Let us extend the resolution to multi-layered media.

The general solution of the diffusion equation in medium $i$, without source term, is:

$$
\widetilde{\varphi}(z)=A_{i} \exp \left(-S_{i} z\right)+B_{i} \exp \left(s_{i} z\right)
$$

where:

$$
s_{i}=\sqrt{k^{2}+\frac{\mu_{a i}}{D_{i}}+\frac{j \omega}{D_{i} c_{i}}}
$$

The boundary conditions (2) become:

$$
\left\{\begin{array}{c}
n_{i+1}^{2}\left[A_{i} e^{-s_{i} l_{i}}+B_{i} e^{s_{i} l_{i}}\right]=n_{i}^{2}\left[A_{i+1} e^{-s_{i+1} l_{i}}+B_{i+1} e^{s_{i+1} l_{i}}\right] \\
D_{i} s_{i}\left[-A_{i} e^{-s_{i} l_{i}}+B_{i} e^{s_{i} l_{i}}\right]=D_{i+1} s_{i+1}\left[-A_{i+1} e^{-s_{i+1} l_{i}}+B_{i+1} e^{s_{i+1} l_{i}}\right]
\end{array}\right.
$$

which can be written with the following form:

$$
\left(\begin{array}{c}
A_{i} \\
B_{i}
\end{array}\right)=P_{i}\left(\begin{array}{c}
A_{i+1} \\
B_{i+1}
\end{array}\right)
$$

where $P_{i}$ is a $2 \times 2$ matrix depending on the optical coefficients.

Consequently:

$$
\left(\begin{array}{l}
A_{1} \\
B_{1}
\end{array}\right)=P_{1} P_{2} P_{3} \ldots P_{N-1}\left(\begin{array}{l}
A_{N} \\
B_{N}
\end{array}\right)
$$

where $B_{N}=0$ to respect the condition $\varphi \rightarrow 0$ when $z \rightarrow \infty$.

To include the source term, one only has ${ }^{[1]}$ to write $\widetilde{\varphi}(z)=A_{0} \exp \left(-s_{1} z\right)+B_{0} \exp \left(s_{1} z\right)$ for $z<z_{0}$ and $\widetilde{\varphi}(z)=A_{1} \exp \left(-s_{1} z\right)+B_{1} \exp \left(s_{1} z\right)$ for $\mathrm{z}_{0}<\mathrm{z}<l_{1}$ with the boundary conditions:

$$
\begin{aligned}
& \widetilde{\varphi}\left(z_{0}^{+}\right)=\widetilde{\varphi}\left(z_{0}^{-}\right) \\
& \frac{\partial \widetilde{\varphi}}{\partial z}\left(z_{0}^{+}\right)-\frac{\partial \widetilde{\varphi}}{\partial z}\left(z_{0}^{-}\right)=-1 / D_{1}
\end{aligned}
$$

which gives a linear relationship between $A_{0}, B_{0}$ and $A_{1}, B_{1}$. The knowledge of $A_{N}$ therefore gives the solution of the problem in all layers (Appendix A). 
The Extrapolated Boundary Condition (3) which can be rewritten $\varphi\left(-z_{b}\right)=A_{0} \exp \left(s_{1} z_{b}\right)+B_{0} \exp \left(-s_{1} z_{b}\right)=0, \quad$ eventually allows to get $A_{N}$. The reflectance function can then be deduced from ${ }^{[8]}$.

$$
\widetilde{R}\left(\omega, k^{2}\right)=-j_{z}(0)=D_{1} \frac{\partial \widetilde{\varphi}}{\partial z}(0)
$$

and from an inverse Fourier transform, using the $J_{0}$ Bessel function:

$$
R(\vec{\rho}, t)=\frac{1}{4 \pi^{2}} \int_{0}^{\infty} k d k \int d \omega \widetilde{R}\left(\omega, k^{2}\right) J_{0}(k \rho) \exp (j \omega t)
$$

We have here a quite general formula. The particular case of a two-layered media with $n_{1}=n_{2}$ can be solved using ${ }^{[1]}$ :

$$
\widetilde{R}=\operatorname{ch}\left[s_{1} z_{b}\right] \frac{D_{1} s_{1} \operatorname{ch}\left[s_{1}\left(l-z_{0}\right)\right]+D_{2} s_{2} \operatorname{sh}\left[s_{1}\left(l-z_{0}\right)\right]}{D_{1} s_{1} \operatorname{ch}\left[s_{1}\left(l+z_{b}\right)\right]+D_{2} s_{2} \operatorname{sh}\left[s_{1}\left(l+z_{b}\right)\right]}
$$

\section{3)- Asymptotic behavior of the reflectance function at infinite times.}

A numerical evaluation of (9) is not easy to perform, but its asymptotic behavior can be obtained from the saddle point method. Let us use in (9) the substitution $\omega \mapsto s=s_{N}$. We have at fixed k: $j \omega=D_{N} c_{N}\left(s^{2}-k^{2}\right)-\mu_{a_{N}} c_{N}$ and $d \omega=-2 j D_{N} c_{N} s d s$. Using these expressions in (9) leads directly to :

$$
R(\vec{\rho}, t)=\frac{-j D_{N} c_{N}}{2 \pi^{2}} \exp \left(-\mu_{a N} c_{N} t\right) \int_{0}^{\infty} k d k \int_{\Gamma(k)} s d s \widetilde{R}\left(s, k^{2}\right) J_{0}(k \rho) \exp \left(D_{N} c_{N}\left[s^{2}-k^{2}\right] t\right)
$$

where $\Gamma(\mathrm{k})$ is the hyperbolic contour $s(\omega)$ in the complex plane at a given $\mathrm{k}$, for $\omega$ varying from $-\infty$ to $\infty$. This choice for s leads to a privileged role for the optical coefficients of the deepest layer, as can be seen for instance from the $\exp \left(-\mu_{a_{N}} c_{N} t\right)$ term. The reasons for this choice are developed in appendix B. The only point we will stress here is that with this choice, $\widetilde{R}\left(s, k^{2}\right)$ is an analytic function of s. From this fact, it appears than the integration on 
$\Gamma(\mathrm{k})$ can be replaced by an integration on $] 0^{+}-j \infty, 0^{+}+j \infty\left[\right.$, providing $\widetilde{R}\left(s, k^{2}\right)$ has no poles inside the contour defined in figure 2. On this integration axis the integrand in (10) is proportional to the Gaussian $\exp \left(s^{2} t / \gamma\right)$ which is all the more peaked at $s=0$ than $\mathrm{t}$ is high. It is therefore justified to use the following development:

$$
\widetilde{R}\left(s, k^{2}\right)=\sum_{p, q \geq 0} \widetilde{R}_{p, q} s^{p} k^{2 q}
$$

In the following we will note $\mu_{a}, D$ and $c$ instead of $\mu_{a N}, D_{N}$ and $c_{N}$. Inserting (11) in (10) and integrating on $] 0^{+}-j \infty, 0^{+}+j \infty[$ leads to:

$$
\begin{aligned}
R(\vec{\rho}, t)=-D c \frac{\sqrt{2 \pi}}{2 \pi^{2}}\left\{\sum_{n \geq 2} \widetilde{R}_{2 p+1, q \geq 0}\right. & \left.(2 p+1) ! !(2 D c t)^{-p}(-1)^{p+q} \Delta_{T}^{q}+O\left(t^{-n-1}\right)\right\} \times \\
& \times(2 D c t)^{-\frac{5}{2}} \exp \left[-\frac{\rho^{2}}{4 D c t}-\mu_{a} c t\right]
\end{aligned}
$$

where $\Delta_{\mathrm{T}}$ is the transverse laplacian. From this expression we deduce:

$$
R(\vec{\rho}, t)=\left[\sum_{n \geq p \geq 2 q \geq 0} R_{p, q} \rho^{2 q} t^{-p}+O\left(t^{-n-1}\right)\right] t^{-5 / 2} \exp \left[-\frac{\rho^{2}}{4 D c t}-\mu_{a} c t\right]
$$

or

$$
\ln R(\vec{\rho}, t)=-\frac{5}{2} \ln t-\frac{\rho^{2}}{4 D c t}-\mu_{a} c t+\sum_{n \geq p \geq 2 q \geq 0} C_{p, q} \rho^{2 q} t^{-p}+O\left(t^{-n-1}\right)
$$

At long times the reflectance function therefore converges toward the solution of a semiinfinite geometry, with the optical coefficients of the deepest layer. This development is similar to the result obtained by Mochi et $\mathrm{al}^{[14]}$ in two dimension with $\rho=0$.

An example is presented in figure 3 to illustrate the convergence of (12), using the medium described in table 1 , with $l=1 \mathrm{~cm}$ and with $\rho=0 . \ln R_{\infty}-\ln R_{n} \approx \Delta R / R$ is plotted versus time, for different values of the order $n$ of the development, and using a logarithmic scale. As can be seen in this figure, the order $n=0$ leads to more than $10 \%$ error for $\mathrm{t}<9 \mathrm{~ns}$, the 
order $n=1$ is over this value for $\mathrm{t}<3$ ns. As the physical accessible values rather lie between 0 and $3 \mathrm{~ns}$, some refinements are needed in order to exploit (13).

\section{4)- Behavior of the reflectance function at practical "long times"}

In practical situations, the asymptotic behavior is analyzed in some interval around a relatively high value $t_{0}$. In that way we need to access to a development around $t_{0}$, which can slightly differ from (13). To analyze this, let us set $s=j \theta$ in (10). Taking into account that $\widetilde{R}\left(0, k^{2}\right)$ gives no contribution in (10), so that $\widetilde{R}\left(s, k^{2}\right)$ can be replaced by $\widetilde{R}\left(s, k^{2}\right)-\widetilde{R}\left(0, k^{2}\right)$, we arrive to (Appendix C):

$$
\begin{aligned}
R(\rho, t)=-\alpha^{5 / 2} & \pi^{-5 / 2} \exp \left[-\alpha \rho^{2}-\mu_{a} c t\right] \times \\
& \times \int_{0}^{\infty} \mathrm{R} d \mathrm{R} \int_{-\infty}^{\infty} d \mathrm{Z} \hat{R}(\mathrm{Z}, \mathrm{R})\left(1-2 \alpha \mathrm{Z}^{2}\right) J_{0}(2 j \alpha \rho \mathrm{R}) \exp \left[-\alpha\left(\mathrm{R}^{2}+\mathrm{Z}^{2}\right)\right]
\end{aligned}
$$

where $\alpha=1 /(4 D c t)$ and where:

$$
\hat{R}(\mathrm{Z}, \mathrm{R})=\int d \theta d^{2} k \frac{D c}{j \pi \theta}\left[\widetilde{R}\left(s, k^{2}\right)-\widetilde{R}\left(0, k^{2}\right)\right] \exp [j \vec{k} \cdot \overrightarrow{\mathrm{R}}+j \mathrm{Z} \theta]
$$

Setting $\alpha=\alpha_{0}+\delta \alpha$ in the integrand of (14) leads to a development in $\rho^{q} \delta \alpha^{p}$ which is very similar to (12). Let us explicit this development to the first order:

$$
\begin{gathered}
R(\rho, t)=-\alpha^{5 / 2} \pi^{-5 / 2} \exp \left[-\alpha \rho^{2}-\mu_{a} c t\right] \int \mathrm{R} d \mathrm{R} d \mathrm{Z} \hat{R}(\mathrm{Z}, \mathrm{R}) \exp \left[-\alpha_{0}\left(\mathrm{R}^{2}+\mathrm{Z}^{2}\right)\right] \times \\
\times\left\{\left[1-2 \alpha_{0} \mathrm{Z}^{2}\right]-\left[2 \mathrm{Z}^{2}+\left(1-2 \alpha_{0} \mathrm{Z}^{2}\right)\left(\mathrm{R}^{2}+\mathrm{Z}^{2}\right)\right] \delta \alpha+\mathrm{R}^{2}\left[1-2 \alpha_{0} \mathrm{Z}^{2}\right] \alpha^{2} \rho^{2}\right. \\
\left.-\mathrm{R}^{2}\left[2 \mathrm{Z}^{2}+\left(1-2 \alpha_{0} \mathrm{Z}^{2}\right)\left(\mathrm{R}^{2}+\mathrm{Z}^{2}\right)\right] \alpha_{0}^{2} \rho^{2} \delta \alpha\right\}
\end{gathered}
$$

This expression differs from (12) only through the last term, which has the form $\rho^{2} / t$ and can influence the result obtained for the diffusion coefficient D. We however experienced that this term can be neglected if, firstly, $\hat{R}$ is sufficiently peaked to 0 , and secondly, if $\alpha_{0}$ is small enough. The first condition requires that there is no pole of $\widetilde{R}\left(s, k^{2}\right)$ in the vicinity of 
the pure imaginary axis, in order for $\widetilde{R}$ to be relatively smooth on this axis, and for $\hat{R}$ to be a peaked function. In order to fulfill the second condition, one has to chose $t_{0}$ as high as possible with a reasonable signal to noise ratio. The validity domain of this approximation will be discussed in section 8 . Starting from this hypothesis and taking the logarithm of (15) up to the first order leads to:

$$
\ln R(\vec{\rho}, t) \approx R_{0}-\frac{5}{2} \ln t-\frac{\rho^{2}}{4 D c t}-\mu_{a} c t+\frac{E}{t}+F \frac{\rho^{2}}{t^{2}}
$$

The parameters E and F calculated from (15) are represented in figure 4a-b) versus $t_{0}=1 /\left(4 D c \alpha_{0}\right)$, in the case of the medium of table 1 with $l=1 \mathrm{~cm}$. The corresponding values $C_{1,0}$ and $C_{2,1}$ of the development (13) are shown with dashed lines. One can see in figure $4-a)$ that in the $1 . .3 \mathrm{~ns}$ range, the value of $\mathrm{E}$ is significantly different from its asymptotic value $C_{1,0}$. The coefficient $\mathrm{F}$ in figure 4-b) is less different from $C_{2,1}$.

One way to obtain the optical coefficients of the deepest layer using experimental results obtained for a space domain near $\rho=0$ and a time domain near a chosen time $t_{0}$ is to fit the logarithm of the reflectance function using (16), with five adjustable parameters that are $R_{0}, \mu_{a} c, D c, E$ and $F$. From the result of this fit one can extract $\mu_{a}$ and $\mu_{s}^{\prime}$.

\section{5)- Implementation of the inverse problem.}

The logarithm of the reflectance function in equation (16) depends linearly on the parameters, and the inverse problem reduces to a simple linear regression: If $\boldsymbol{y}$ is a vector of size $\mathrm{M}$ collecting all the experimental data for different positions in time and space, $\boldsymbol{p}$ a vector which represents the 5 parameters, we can write $\boldsymbol{y} \sim \boldsymbol{J} \boldsymbol{p}$ ( where the Jacobian $\boldsymbol{J}$ is defined by $\left.J_{i j}=\partial y_{i} / \partial p_{j}\right)$ and the variance 


$$
\sigma^{2}=M^{-1}\|\boldsymbol{y}-\boldsymbol{J p}\|^{2}
$$

is minimized by

$$
\boldsymbol{p}=\left[{ }^{t} \boldsymbol{J} \boldsymbol{J}\right]^{-1}{ }^{t} \boldsymbol{J} \boldsymbol{y}
$$

The inverse problem can therefore be solved through a simple matrix inversion, leading to a unique result, without the problem of the choice of initial values. If we assume that the variance is almost constant on the small region considered for the linear regression (i.e. $<\delta \boldsymbol{y}^{t} \delta \boldsymbol{y}>=\sigma^{2} \boldsymbol{I}$, with $\boldsymbol{y}=\boldsymbol{y}_{0}+\delta \boldsymbol{y}, \boldsymbol{p}=\boldsymbol{p}_{0}+\delta \boldsymbol{p}$ and $\boldsymbol{y}_{0}=\boldsymbol{J} \boldsymbol{p}_{\boldsymbol{0}}$ ), the covariance matrix reads:

$$
<\delta \boldsymbol{p}^{t} \delta \boldsymbol{p}>=\sigma^{2}\left[{ }^{t} \boldsymbol{J} \boldsymbol{J}\right]^{-1}
$$

and the square root of the diagonal elements of the covariance matrix corresponds to the average statistical error on the different parameters. One can also define the correlation between two parameters with $\operatorname{Corr}\left(p_{i}, p_{j}\right)=<\delta p_{i} \delta p_{j}>/\left[<\delta p_{i}^{2}><\delta p_{j}^{2}>\right]^{1 / 2}$.

In order to test our inversion procedure, we numerically evaluate some reflectance functions with a Monte Carlo simulator ${ }^{[16]}$, using a Henyey-Greenstein phase function with an anisotropy factor $g=0.8$. The random walkers are collected at a distance $r$ and at time $t$ in a ring of size $\Delta \rho=137 \mu \mathrm{m}$ and $\Delta t=20 \mathrm{ps}$. The result is then normalized to 1 incident photon, and to a pixel size of $\Delta \rho \times \Delta \rho \times \Delta t$. The fits were performed for $1.14 \mathrm{~cm}<\rho<2.49 \mathrm{~cm}$, what corresponds to an array of 100 detectors of size $\Delta \rho \times \Delta \rho$, and for 1500ps $<t<2000 p s$ ( i.e. $t_{0}=1750 \mathrm{ps}$ ), with a time resolution of $20 \mathrm{ps}$. Such a setup can correspond to the use of a streak camera.

Let us begin with the medium described in table 1, which can be used to describe the neonate head ${ }^{[15]}$. We present on figure 5 a part of the result obtained from the Monte Carlo simulation for a thickness $l=5 \mathrm{~mm}$ for the first layer. We plot on this figure the logarithm of the time-resolved reflectance function corresponding to 6 detectors chosen with an equal 
spacing in $[1.14 \mathrm{~cm}, 2.49 \mathrm{~cm}]$ (detectors $n^{\circ} 0,20,40,60,80$ and100). The highest curve obviously corresponds to the detector placed at $\rho=1.14 \mathrm{~cm}$. We represent too the fit performed for $1500 \mathrm{ps}<t<2000 \mathrm{ps}$, together with a zoom of this time interval. The 6 curves look like straight lines in this small region: as a line is described by two parameters, and as these parameters depend on the position $\rho$, one can await at least 4 parameters from this set of curves. The fifth parameter comes from the little curvature of these curves. Of course the statistical error on parameters $\mathrm{E}$ and $\mathrm{F}$ can be relatively important, but as these parameters have only a corrective effect on the values of the optical coefficients, this procedure can lead to a reasonable statistical error for the absorption and reduced scattering coefficients. The result presented in figure 5 was obtained using $10^{9}$ random walkers. The link between this number and the signal to noise ratio is however not trivial, as many technical procedure are implied in order to improve this ratio ${ }^{[16]}$. We measure a standard deviation $\sigma=1.51 \%$ for the logarithm of the reflectance function when using our model ( we have $\sigma=1.54 \%$ with the semi-infinite model ). This corresponds to a signal to noise ratio of 65 for the reflectance function $(R / \delta R \sim 1 / \delta \ln R)$. As one needs to detect $65^{2} \sim 4000$ photons in order to have such a signal to noise ratio, and as the average attenuation factor is of about $10^{-10}$, we deduce that $4.10^{13}$ photons are needed for the source. If furthermore the numerical aperture of the detection is limited, for instance with a ratio aperture of 4 , this number has to be increased to $5.10^{15}$ photons, what corresponds to about an irradiation of about $1 \mathrm{~mW}$ with a 1 second onchip integration time. The numerical simulations presented here are therefore compatible with realistic experimental conditions.

The results obtained for $\mu_{a}$ and $\mu_{s}$ for different values of $l$ are shown on figure $6-\mathrm{a}, \mathrm{b}$ ). The squares correspond to the results obtained using a simple semi-infinite fit ( $E=F=0$ ), and the circles correspond to the results obtained using (16). As the semi-infinite fitting procedure requires only 3 parameters, the statistical error is very low and the error bars won't 
be represented in that case. We obtained a clear improvement of the accuracy using our method. The parameters $\mu_{a}$ and $\mu_{s}^{\prime}$ are weakly correlated, when $\mu_{a}$ is highly correlated to $\mathrm{E}$ and $\mu_{s}^{\prime}$ to $\mathrm{F}$ (we have for instance for $l=5 \mathrm{~mm}: \operatorname{Corr}\left(\mu_{a}, \mu_{s}^{\prime}\right)=0.16, \operatorname{Corr}\left(\mu_{a}, E\right)=-0.98$, $\left.\operatorname{Corr}\left(\mu_{s}^{\prime}, F\right)=0.99, \operatorname{Corr}\left(\mu_{a}, F\right)=0.16, \operatorname{Corr}\left(\mu_{s}^{\prime}, E\right)=-0.3\right)$. This high correlation between $\mu_{a}$ , $E$ and $\mu_{s}^{\prime}, F$ is of course not in contradiction with the fact that $E$ and $F$ have only a corrective effect on the values of the optical coefficients The values for $E$ and $F$, obtained from a fit that assumes the correct values for $\mu_{a}$ and $\mu_{s}^{\prime}$, are presented on figure $7-\mathrm{a}, \mathrm{b}$ ) (circles), and compared to the values calculated from (15). We observe a complete agreement for $E$, and a small systematic error for $F$. The deviation from theory comes from the large time window used for the fit. Reducing this window however introduce fatal undesirable noise.

We reproduce this numerical experiment using another two-layered medium described in table 2. The only change concerns the absorption coefficient, which is now lower in the upper layer than in the deepest one. This case approaches the situation of the fat/muscle succession ( the scattering coefficients of fat and muscle are in fact estimated ${ }^{[17]}$ to be lower than the values in table 2, but the case considered here is less advantageous for our method ). The results obtained for $\mu_{a}$ and $\mu_{s}^{\prime}$ are shown on figure 8-a,b), and the values for $E$ and $F$ on figure 9-a,b). Here again we observe an improvement when using our method, but the error increases very quickly when $l>4 m m$. A jump appears on theoretical curves in figure 9 after $l$ $>5 \mathrm{~mm}$, so that for higher thickness the theory completely disagrees with fitted values. This behavior is typically linked to the presence of a pole in the contour of figure 2 . 


\section{6)- Influence of poles.}

Poles account for diffusion waves guided in the layered structure (Appendix A). They induce crucial modifications in the change of the integration contour in equation (10). If $s_{P i}$ denote the poles lying inside the contour of figure 2, one can write:

$$
\int_{\Gamma(k)} s d s \widetilde{R}\left(s, k^{2}\right) \exp \left(D c s^{2}\right)=\int_{-j \infty+0^{+}}^{j \infty+0^{+}} s d s \widetilde{R}\left(s, k^{2}\right) \exp \left(D c s^{2}\right)+2 \pi j \sum_{s_{P i}} \mathscr{K}_{e s} \widetilde{R}\left(s_{P i}, k^{2}\right) \exp \left(D c s_{P i}^{2} t\right)
$$

where $\mathscr{K}_{e s} \widetilde{R}\left(s_{P i}, k^{2}\right)$ stands for the residue of function $\widetilde{R}\left(s, k^{2}\right)$ at $s_{P i}$.

In the example of the medium described by table 2 , a pole appears on the real axis inside the contour of figure 2 when the thickness $l$ of the first slab passes a critical value. In fact, the pole crosses the contour for (Appendix D):

$$
l_{c}+z_{b}=\frac{\pi}{2} \sqrt{\frac{D_{1}}{\mu_{a 2}-\mu_{a 1}}}
$$

Note that this implies $\mu_{a 1}<\mu_{a 2}$, which is the particularity of table 2 . This numerically gives $l_{c}=5.4 \mathrm{~mm}$, which corresponds to the transition length observed in the previous section. In fact we observe in figure 8 and 9 a decrease of the accuracy even for $l<l_{c}$, due to the fact that the pole approaches the imaginary axis so that approximations in (15),(16) are no more valid. This pole can be written:

$$
s_{P}=s_{0}+\beta k^{2}+o\left(k^{2}\right) \text { or } s_{P}^{2}=s_{0}^{2}+2 s_{0} \beta k^{2}+o^{\prime}\left(k^{2}\right)
$$

A rough approximation of $s_{0}$ and $\beta$ is (Appendix D):

$$
s_{0} \approx \frac{\pi^{2} D_{1}}{4\left(l_{c}+z_{b}\right)^{2} D_{2}}\left(l-l_{c}\right) \quad \text { and } \quad \beta \approx \frac{D_{2}-D_{1}}{2 D_{2}}\left(l_{c}+z_{b}\right) .
$$


The point is that $s_{0}>0$, so that the exponential term in the right member of (17) is diverging when increasing time, and the residue is in fact the dominant term:

$$
\int_{\epsilon_{k}} s d s \widetilde{R}\left(s, k^{2}\right) \exp \left(D c s^{2} t\right) \approx 2 \pi j \mathscr{K}_{e s} \cdot \widetilde{R}\left(s_{P}, k^{2}\right) \exp \left(D c s_{P}^{2} t\right)
$$

Reporting this approximation in (10) leads to:

$$
R(\vec{\rho}, t)=\frac{D c}{\pi} \exp \left(-\mu_{a} c t\right) \int_{0}^{\infty} k d k \mathscr{K}_{e s} \widetilde{R}\left(k^{2}\right) \exp \left(D c o^{\prime}\left(k^{2}\right) t\right) J_{0}(k \rho) \exp \left(D c\left[s_{0}^{2}+\left(2 \beta s_{0}-1\right) k^{2}\right] t\right)
$$

Here again the Gaussian term becomes very thin at high times so that we can approximate: $\mathscr{K}_{e s s} \widetilde{R}\left(k^{2}\right) \exp \left(o^{\prime}\left(k^{2}\right) t\right) \approx \mathscr{K}_{e s} \widetilde{R}(0) \exp \left(o^{\prime}(0) t\right)$, leading to :

$$
R(\vec{\rho}, t) \propto \frac{1}{t} \exp \left(-\left[\mu_{a}-D s_{0}^{2}\right] c t-\frac{\rho^{2}}{4\left(1-2 \beta s_{0}\right) D c t}\right)
$$

We therefore have:

$$
\ln R(\rho, t)=R_{0}-\ln t-\mu_{e f f} c t-\frac{\rho^{2}}{4 D_{e f f} c t}
$$

with

$$
\left\{\begin{array}{c}
\mu_{e f f}=\mu_{a}+D s_{0}^{2} \\
D_{e f f}=D\left(1-2 \beta s_{0}\right)
\end{array}\right.
$$

We see in (21) that the $5 / 2$ factor before $\ln t$ disappears, and that the value of the absorption coefficient and diffusion constant are somewhat modified. We present in figure 10a,b) the results obtained on the medium of table 2 for the effective coefficients $\mu_{\text {eff }}$ and $\mu_{\text {seff }}^{\prime}=1 / 3 D_{\text {eff }}$ using (21), together with the values for $\mu_{a}$ and $\mu_{s}^{\prime}$ deduced using (22). We find that (22) allows a relatively good agreement, using however an a priori knowledge of the medium through the values of $s_{0}$ and $\beta$. 


\section{7)- An example with a multi-layered media.}

The considerations on the reflectance function developed in all this paper are not limited to two-layered media, and can be applied to multi-layered structure with the same limitations concerning the position of the poles of the reflectance function. We present here a test of our procedure performed on the 4-layered media described in table 3 . We use in this table the data of table 1 corresponding to the neonate head, and add one layer for the $\operatorname{skin}^{[18]}$ and one layer for the cerebrospinal fluid (CSF). The results obtained with our procedure are presented in table 4 for 3 different values of the absorption coefficient of the deepest layer, and are compared to the results obtained with a simple semi-infinite fit. Our method leads to a significant improvement of the accuracy, for both the absorption and the reduced scattering coefficients. These performances will be of course deteriorated when increasing the thickness of the different layers, depending on the complex behavior of the poles of the reflectance function.

\section{8)- Discussion and Conclusion.}

We have shown in this paper that it is possible to deduce with a high accuracy the optical coefficients of an organ covered by scattering layers from time and space resolved reflectance measurements, provided the thickness of the upper layers is not too high. The case of thicker covering layers was also analyzed; in that case we prove that the asymptotic behavior of the reflectance function is poorly correlated to the properties of the deepest layer, since the former layers behave as wave guides. Therefore, additional information about the encapsulating medium is needed in that regime to understand the asymptotic behavior. 
A criterion giving a limit between these two regimes was given in the case of a twolayered medium, but of course it will be very hard to generalize such criterion to multilayered media. The simplest method in order to determine the regime corresponding to a given medium is to test the inversion method proposed in this paper on simulated data. Such tests will allow the determination of regions in which our method is applicable. The only knowledge then needed is the belonging of the biological medium to this region. The optical properties of the deepest layer of such a medium can then be deduced without any additional information.

Much more work is therefore needed now in order to exploit this method in biomedical applications. One can process as explained in the paragraph above, or can use another investigation method, like the systematic use of this method with several patients, at several locations for each patient, and to study the reproducibility of the results depending on the patient and on the location of the sensor. An interesting point would be to have a discrimination test, relying for instance on the use of different values for $t_{0}$, but this is beyond the scope of this paper. 


\section{$\underline{\text { Appendix A }}$}

Let us set:

$$
P_{1} P_{2} P_{3} \ldots P_{N}=\left(\begin{array}{ll}
a & b \\
c & d
\end{array}\right)
$$

Equation (6) can the be rewritten, taking into account $B_{N}=0$ :

$$
\left\{\begin{array}{l}
A_{1}=a A_{N} \\
B_{1}=c A_{N}
\end{array}\right.
$$

Equation (7) directly leads to:

$$
\left\{\begin{array}{c}
A_{0}=A_{1}-\frac{\exp \left(s_{1} z_{0}\right)}{2 s_{1} D_{1}}=a A_{N}-\frac{\exp \left(s_{1} z_{0}\right)}{2 s_{1} D_{1}} \\
B_{0}=B_{1}+\frac{\exp \left(-s_{1} z_{0}\right)}{2 s_{1} D_{1}}=c A_{N}+\frac{\exp \left(-s_{1} z_{0}\right)}{2 s_{1} D_{1}}
\end{array}\right.
$$

The Extrapolated Boundary Condition (3) then gives:

$$
\left[a \exp \left(s_{1} z_{b}\right)+c \exp \left(-s_{1} z_{b}\right)\right] A_{N} \equiv \operatorname{Den}\left(s, k^{2}\right) A_{N}=\frac{\operatorname{sh}\left[s_{1}\left(z_{0}+z_{b}\right)\right]}{s_{1} D_{1}}
$$

and we finally have:

$$
\widetilde{R}\left(s, k^{2}\right)=-s_{1} D_{1}\left[A_{0}-B_{0}\right]=\frac{a \exp \left(-s_{1} z_{0}\right)+c \exp \left(s_{1} z_{0}\right)}{\operatorname{Den}\left(s, k^{2}\right)} \operatorname{ch}\left(s_{1} z_{b}\right)
$$

If we cancel the source term by setting (A1) equal to 0 , we can have non trivial solutions if $\operatorname{Den}\left(s, k^{2}\right)=0$; from (A2), such guided waves solutions corresponds to the poles of $\widetilde{R}$. 


\section{$\underline{\text { Appendix B }}$}

To begin with, we notice that the resolution procedure for $\widetilde{R}$ (section 2 ) only involves exponential functions and resolutions of linear systems, so that $\widetilde{R}$ is an analytic function of $s_{1}$, $s_{2}, \ldots, s_{N}$. The point now is that for any layer $i<N$, the substitution $s_{i} \mapsto-s_{i}$ corresponds to the substitution $A_{i} \leftrightarrow B_{i}$. This substitution has no other influence on the equations, and leaves $\widetilde{R}$ invariant. So $\widetilde{R}$ is an even function of $s_{1}, s_{2}, \ldots, s_{N-1}$, and is therefore an analytic function of $s_{1}^{2}, \ldots, s_{N-1}^{2}$. However $\widetilde{R}$ is not an even function of $s_{N}$.

Let us consider the more general substitution $\omega \mapsto s=\sqrt{\eta+j \gamma \omega}$ at fixed $\mathrm{k}$, where $\eta$ and $\gamma$ are positive real numbers. We have instead of (10):

$$
R(\vec{\rho}, t)=\frac{-j}{2 \gamma \pi^{2}} \int_{0}^{\infty} k d k \int_{\Gamma(\eta)} s d s \widetilde{R}\left(s, k^{2}\right) J_{0}(k \rho) \exp \left(\left[s^{2}-\eta\right] t / \gamma\right)
$$

As $s_{i}^{2}=k^{2}+\frac{\mu_{a_{i}}}{D_{i}}+\frac{s^{2}-\eta}{\gamma D_{i} c_{i}}$ are obviously analytic functions of $s$, the only thing to do for ensuring the analyticity of $\widetilde{R}\left(s, k^{2}\right)$ is to check the analyticity of $s_{N}=\sqrt{\left(s^{2}+\sigma\right) /\left(\gamma D_{N} c_{N}\right)}$, where $\sigma=\gamma c_{N}\left[D_{N} k^{2}+\mu_{a N}\right]-\eta$ is real.

If the square root function is defined for complex numbers with an argument in $]-\pi, \pi$, that is with a cut in the complex plane located on the real axis at $]-\infty, 0\left[, s_{N}\right.$ is analytic in the half plane $\Re_{e} s>0$ for $\sigma \geq 0$, and the integration on $\Gamma(\mathrm{k})$ can be replaced by an integration on $] 0^{+}-j \infty, 0^{+}+j \infty\left[\right.$. But if $\sigma>0$, we have $s_{N}(s)=s_{N}(-s)$ for any $s$ on $\left.\mid 0^{+}-j \sqrt{\sigma}, 0^{+}+j \sqrt{\sigma}\right] ; \quad \widetilde{R}$ is then an even function of $s$ and (B1) is zero on that segment, leading for any $\mathrm{n}>0$ to: 


$$
R(\vec{\rho}, t)=O_{\rho}\left(t^{-n}\right) \exp \left[-\left(\mu_{a}-\delta \mu\right) t\right] \quad(\delta \mu>0)
$$

This is not astonishing, as $\exp [-\delta \mu t]=O\left(t^{-n}\right)$, but this is useless. So we need to choose $\sigma=0$, that is $s=\left(\sqrt{\gamma D_{N} c_{N}}\right) s_{N}=s_{N}$ (since $\gamma$ is a simple scaling factor which can be set as $\left.\gamma=1 / D_{N} c_{N}\right)$ and the deepest layer plays then a central role, as awaited. 


\section{Appendix C}

We have already seen that $\widetilde{R}\left(s, k^{2}\right)$ can be replaced by $\widetilde{R}\left(s, k^{2}\right)-\widetilde{R}\left(0, k^{2}\right)$ in $(10)$. Let us rewrite (10) using an integration over the whole $\boldsymbol{k}$ plane (without using the cylindrical symmetry), and insert $\int \delta\left(\theta-\theta^{\prime}\right) \delta\left(\boldsymbol{k}-\boldsymbol{k}^{\prime}\right) d \theta^{\prime} d^{2} k^{\prime}$; we obtain:

$$
\begin{aligned}
& \begin{array}{r}
R(\rho, t)=\frac{-D c}{4 \pi^{3}} \exp \left(-\mu_{a} c t\right) \int d^{2} k d^{2} k^{\prime} d \theta d \theta^{\prime} s^{-1}\left[\widetilde{R}\left(s, k^{2}\right)-\widetilde{R}\left(0, k^{2}\right)\right] \times \\
\quad \times \delta\left(\theta-\theta^{\prime}\right) \delta\left(\boldsymbol{k}-\boldsymbol{k}^{\prime}\right) \theta^{\prime 2} \exp \left(-D c\left[\theta^{\prime 2}+k^{\prime 2}\right] t-j \boldsymbol{k}^{\prime} \cdot \boldsymbol{\rho}\right)
\end{array} \\
& \text { using } \delta\left(\theta-\theta^{\prime}\right) \delta\left(\boldsymbol{k}-\boldsymbol{k}^{\prime}\right)=\frac{1}{8 \pi^{3}} \iint \exp \left[j \mathrm{Z}\left(\theta-\theta^{\prime}\right)+j \mathbf{R} \cdot\left(\boldsymbol{k}-\boldsymbol{k}^{\prime}\right)\right] d Z d^{2} \mathrm{R}
\end{aligned}
$$

we get two different contributions:

$$
\int d^{2} k d \theta \frac{1}{j \theta}\left[\widetilde{R}\left(s, k^{2}\right)-\widetilde{R}\left(0, k^{2}\right)\right] \exp [j \mathrm{Z} \theta+j \mathbf{R} \cdot \boldsymbol{k}] \equiv \frac{\pi}{D c} \hat{R}(\mathrm{Z}, \mathrm{R})
$$

and

$$
\int d^{2} k^{\prime} d \theta^{\prime} \theta^{\prime 2} \exp \left[-D c\left[\theta^{\prime 2}+k^{\prime 2}\right] t-j \boldsymbol{k}^{\prime} \cdot(\boldsymbol{\rho}+\mathbf{R})-j \theta^{\prime} \mathrm{Z}\right]
$$

We recognize in this second contribution the Fourier transform of a Gaussian with :

$$
\begin{aligned}
& \int d \theta^{\prime} \exp \left[-D c \theta^{\prime 2} t-j \theta^{\prime} \mathrm{Z}\right]=\sqrt{\frac{\pi}{D c t}} \exp \left[-\frac{\mathrm{Z}^{2}}{4 D c t}\right] \\
& \int d \theta^{\prime} \theta^{\prime 2} \exp \left[-D c \theta^{\prime 2} t-j \theta^{\prime} \mathrm{Z}\right]=-\sqrt{\frac{\pi}{D c t}} \frac{d^{2}}{d \mathbf{Z}^{2}} \exp \left[-\frac{\mathrm{Z}^{2}}{4 D c t}\right] \\
& =-\sqrt{\frac{\pi}{D c t}}\left(\frac{\mathrm{Z}^{2}}{(D c t)^{2}}-\frac{1}{2 D c t}\right) \exp \left[-\frac{\mathrm{Z}^{2}}{4 D c t}\right] \\
& \int d k^{\prime 2} \exp \left[-D c k^{\prime 2} t-j \boldsymbol{k}^{\prime} \cdot(\mathbf{R}+\boldsymbol{\rho})\right]=\frac{\pi}{D c t} \exp \left[-\frac{\mathrm{R}^{2}+\rho^{2}+2 \mathbf{R} \cdot \boldsymbol{\rho}}{4 D c t}\right]
\end{aligned}
$$


Notice that the term $\mathbf{R} \cdot \boldsymbol{\rho}$ is the only one that breaks the cylindrical symmetry, so that writing $d^{2} \mathrm{R}=\mathrm{R} d \mathrm{R} d \varphi$ and $\mathbf{R} \cdot \rho=\operatorname{R} \rho \cos \varphi$, we can directly integrate over $\varphi$ :

$$
\int d \varphi \exp \left[j \frac{2 j \mathrm{R} \rho}{4 D c t} \cos \varphi\right]=2 \pi J_{0}\left(\frac{2 j \mathrm{R} \rho}{4 D c t}\right)
$$

Setting $\alpha=\frac{1}{4 D c t}$ and putting all things together leads directly to (14).

\section{$\underline{\text { Appendix D }}$}

Let us consider the poles of :

$$
\widetilde{R}=\operatorname{ch}\left[s_{1} z_{b}\right] \frac{D_{1} s_{1} \operatorname{ch}\left[s_{1}\left(l-z_{0}\right)\right]+D_{2} s_{2} \operatorname{sh}\left[s_{1}\left(l-z_{0}\right)\right]}{D_{1} s_{1} \operatorname{ch}\left[s_{1}\left(l+z_{b}\right)\right]+D_{2} s_{2} \operatorname{sh}\left[s_{1}\left(l+z_{b}\right)\right]}
$$

Writing $l^{*}=l+z_{b}$, theses poles satisfy:

$$
\operatorname{cth}\left[s_{1}\left(s_{P}\right) l^{*}\right]=-\frac{D_{2} s_{P}}{D_{1} s_{1}\left(s_{P}\right)}
$$

where we recall $s_{1}\left(s_{P}\right)=\sqrt{\frac{D_{2}}{D_{1}} s_{p}^{2}+k^{2}\left(1-\frac{D_{2}}{D_{1}}\right)+\frac{\mu_{a 1}-\mu_{a 2}}{D_{1}}}$.

It appears numerically that a first pole on the real axis crosses the imaginary axis for a critical thickness of the first layer. Let us indeed set $s_{P}=0$ : equation (D1) can have a solution (for $\mathrm{k}=0$ ) only when $\mu_{a 1}<\mu_{a 2}$. We have in that case:

$$
\operatorname{cth}\left[j l_{c}^{*} \sqrt{\frac{\mu_{a 2}-\mu_{a 1}}{D_{1}}}\right]=-j \operatorname{ctg}\left[l_{c}^{*} \sqrt{\frac{\mu_{a 2}-\mu_{a 1}}{D_{1}}}\right]=0
$$


leading to (18). Now if $l^{*}=l_{c}^{*}+\delta l$, we await $s_{P} \approx 0$ and $s_{1}\left(s_{P}\right) \approx s_{1}(0)$ so that (D1) becomes:

$$
-s h^{-2}\left[s_{1}(0) l_{c}^{*}\right] s_{1}(0) \delta l=-\frac{D_{2} s_{P}}{D_{1} s_{1}(0)}
$$

or

$$
s_{P}=\frac{D_{1}}{D_{2}} s_{1}^{2}(0) \delta l=\frac{\pi^{2} D_{1}}{4 l_{c}^{* 2} D_{2}}\left(l-l_{c}\right)
$$

in the same manner we can follow the influence of $\mathrm{k}$ by setting

$$
s_{1}\left(s_{p}\right) \approx s_{1}(0)+\frac{k^{2}}{2 s_{1}(0)}\left(1-\frac{D_{2}}{D_{1}}\right)
$$

and

$$
-s h^{-2}\left[s_{1}(0) l_{c}^{*}\right] \frac{k^{2}}{2 s_{1}(0)}\left(1-\frac{D_{2}}{D_{1}}\right) l_{c}^{*}=-\frac{D_{2} s_{P}}{D_{1} s_{1}(0)} \Rightarrow s_{P}=\frac{k^{2}}{2}\left(1-\frac{D_{1}}{D_{2}}\right) l_{c}^{*}
$$

Corresponding author Jean-Michel Tualle may be reached by email, tualle@galilee.univ-paris13.fr; phone, 33-1-49404092; or fax, 33-1-49403200. 


\section{$\underline{\text { References }}$}

1 A. Kienle, T. Glanzmann, G. Wagnières, H. Van den Bergh, "Investigation of twolayered turbid media with time-resolved reflectance," Applied Optics 37, 68526862 (1998).

2 A. Kienle, M.S. Patterson, N. Dögnitz, R. Bays, G. Wagnières, H. Van den Bergh, "Noninvasive determination of the optical properties of two-layered turbid media," Applied Optics 37, 779-791 (1998).

3 G. Alexandrakis, T.J. Farrell and M.S. Patterson, "Monte carlo diffusion hybrid model for photon migration in a two-layer turbid medium in the frequency domain”, Applied Optics 39, 2235-2244 (2000).

4 G. Alexandrakis, D.R. Busch, G.W. Faris and M.S. Patterson, "Determination of the optical properties of two-layer turbid media by use of a frequency-domain hybrid Monte Carlo diffusion model”, Applied Optics 40, 3810-3821 (2000).

5 J.-M. Tualle, E. Tinet, J. Prat and S. Avrillier, "Light propagation near turbidturbid planar interfaces”, Optics Communications 183, 337-346 (2000).

6 J.-M. Tualle, J. Prat, E. Tinet and S. Avrillier, "Real-space Green's function calculation for the solution of diffusion equation in stratified turbid media", J.O.S.A. A 17, 2046-2055 (2000).

7 A. Pifferi, A. Torricelli, P. Taroni and R. Cubeddu, "Reconstruction of absorber concentrations in a two-layer structure by use of multidistance time-resolved reflectance spectroscopy", Optics Letters 26, 1963-1965 (2001).

8 M.S. Patterson, B. Chance and B.C Wilson, "Time resolved reflectance and transmittance for the non invasive measurement of tissue optical properties", Applied Optics 28, 2331-2336 (1989). 
9 E. Akkermans, P.E. Wolf, and R. Maynard, "Coherent backscattering of light by disordered media: analysis of the peak line shape," Phys. Rev. Lett. 56, 1471-1474 (1986).

10 J. Ripoll and M. Nieto-Vesperinas, "Index mismatch for diffuse photon density waves at both flat and rough diffuse-diffuse interfaces", J. Opt. Soc. Am. A 16, 1947-1957 (1999).

11 R. Aronson, "Boundary conditions for diffusion of light", J. Opt. Soc. Am. A 12 , 2532-2539 (1995).

12 R.C. Haskell, L.O. Svaasand, T.-T. Tsay et al., "Boundary conditions for the diffusion equation in radiative transfer", J. Opt. Soc. Am. A 11, 2727-2741 (1994).

13 A. Kienle and M.S. Patterson, "Improved solutions of the steady-state and the time-resolved diffusion equations for reflectance from a semi-infinite turbid medium", J. Opt. Soc. Am. A 14, 246-254 (1997).

14 M. Mochi, G. Pacelli, M.C. Recchioni et al., "Inverse Problem for a Class of TwoDimensional Diffusion Equations with Piecewise Constant Coefficients," Journal of Optimization Theory and Applications 100, p. 29-57 (1999).

15 See

http://www.medphys.ucl.ac.uk/research/borg/research/NIR topics/phantoms.htm

16 E. Tinet, S. Avrillier, J.-M. Tualle et al., "Fast semi-analytical Monte Carlo simulation for time resolved light propagation in turbid media”, J. Opt. Soc. Am. A 13, 1903-1915 (1996).

17 S. Avrillier, E. Tinet, J-M. Tualle, F. Costes, F. Revel, J-P. Ollivier, "Real-time inversion using Monte Carlo results for the determination of absorption coefficients in multilayered tissues: application to noninvasive muscle oximetry 
(Part 2)" in Diagnostic Optical Spectroscopy in Biomedicine, T.G. Papazoglou and G.A. Wagnieres, eds., Proc. SPIE 4432 , 75-84, (2001).

18 C.R. Simpson, M. Kohl, M. Essenpreis, M. Cope, "Near-infrared optical properties of ex vivo human skin and subcutaneous tissues measured using the Monte Carlo inversion technique“, Phys. Med. Biol. 43, p. 2465-2478, (1998). 


\section{Figure captions}

figure 1: $\quad$ A layered medium.

figure 2: Integration contour. The right curve corresponds to hyperbolic contour $\Gamma(\mathrm{k})$, that is to $\Re_{e}\left(s^{2}\right)=k^{2}+\mu_{a} / D$, the left part is the imaginary axis $] 0^{+}-j \infty, 0^{+}+j \infty$ [. The integration contour is closed by arbitrary upper and lower curves that have no contribution when rejected to infinity.

figure 3: $\quad$ Convergence of the development in equation (12), using the medium described in table 1 with $l=1 \mathrm{~cm}$, at $\rho=0 . \ln \mathrm{R}_{\infty}-\ln \mathrm{R}_{\mathrm{n}}$ is plotted versus time, for different values of the order $n$ of the development, and using a logarithmic scale.

figure 4: $\quad$ Parameters E (fig. a) and F (fig. b) calculated from (15) versus $t_{0}=1 /\left(4 D c \alpha_{0}\right)$, in the case of the medium of table 1 with $l=1 \mathrm{~cm}$. The values $C_{1,0}$ and $C_{2,1}$ of the development (13) are shown in dashed lines.

figure 5: Logarithm of the time-resolved reflectance function obtained from a Monte Carlo simulation with the medium of table 1 and $l=5 \mathrm{~mm}$, for 6 detectors chosen with an equal spacing in $[1.14 \mathrm{~cm}, 2.49 \mathrm{~cm}]$. The fit, performed for $1500 \mathrm{ps}<t<2000 \mathrm{ps}$, is presented together with a zoom of this time interval.

figure 6: $\quad$ Results obtained for $\mu_{a}$ (fig. a) and $\mu_{s}^{\prime}$ (fig. b) for different values of $l$, using the medium described in table 1 . The squares correspond to the results obtained using a 
simple semi-infinite fit ( $E=F=0$ ), and the circles correspond to the results obtained using (16).

figure 7: $\quad$ Results obtained (circles) for E (fig. a) and F (fig. b), assuming correct values for $\mu_{a}$ and $\mu_{s}^{\prime}$, for different values of $l$. The lines with open circles correspond to the results obtained using (16).

figure 8: $\quad$ Results obtained for $\mu_{a}$ (fig. a) and $\mu_{s}^{\prime}$ (fig. b) for different values of $l$, using the medium described in table 2. The squares correspond to the results obtained using a simple semi-infinite fit ( $E=F=0$ ), and the circles correspond to the results obtained using (16).

figure 9: $\quad$ Results obtained (circles) for E (fig. a) and F (fig. b), assuming correct values for $\mu_{a}$ and $\mu_{s}^{\prime}$, for different values of $l$. The lines with open circles correspond to the results obtained using (16)

figure 10: $\quad$ Results obtained (circles) for $\mu_{\text {eff }}$ (fig. a) and $\mu_{\text {seff }}^{\prime}$ (fig. b) using equation (21) on the medium of table 2 , for different values of $l$, together with their values corrected using (22) (squares). 
Table 1: Optical coefficients of a two-layered media for the simulation of a neonate head ${ }^{[15]}$.

\begin{tabular}{|c|c|c|c|}
\hline & $\mu_{s}^{\prime}\left(\mathrm{cm}^{-1}\right)$ & $\mu_{a}\left(\mathrm{~cm}^{-1}\right)$ & $n$ \\
\hline Layer 1 & 15 & 0.15 & 1.4 \\
\hline Layer 2 & 10 & 0.10 & 1.4 \\
\hline
\end{tabular}

Table 1 
Table 2: Optical coefficients of a two-layered media for the simulation of the fat $/$ muscle succession.

\begin{tabular}{|c|c|c|c|}
\hline & $\mu_{s}^{\prime}\left(\mathrm{cm}^{-1}\right)$ & $\mu_{a}\left(\mathrm{~cm}^{-1}\right)$ & $n$ \\
\hline Layer 1 & 15 & 0.026 & 1.4 \\
\hline Layer 2 & 10 & 0.15 & 1.4 \\
\hline
\end{tabular}

Table 2 
Table 3: Optical coefficients of a 4-layered media for the simulation of a neonate head.

\begin{tabular}{|c|c|c|c|c|}
\hline & $\mu_{s}^{\prime}\left(\mathrm{cm}^{-1}\right)$ & $\mu_{a}\left(\mathrm{~cm}^{-1}\right)$ & Thickness (mm) & $n$ \\
\hline Skin & 20 & 0.13 & 2 & 1.45 \\
\hline Skull & 15 & 0.15 & 5 & 1.45 \\
\hline CSF & 0.1 & 0.01 & 0.5 & 1.33 \\
\hline Brain & 10 & $0.05-0.1-0.15$ & - & 1.4 \\
\hline
\end{tabular}

Table 3 
Table 4: Results obtained from simulations performed with the data of table 3.

\begin{tabular}{|c|c|c|c|c|}
\hline \multirow{2}{*}{$\mu_{a, \text { Brain }}\left(\mathrm{cm}^{-1}\right)$} & \multicolumn{2}{|c|}{5 -parameters fit } & \multicolumn{2}{c|}{ Semi-infinite fit } \\
\cline { 2 - 5 } & $\mu_{s}^{\prime}\left(\mathrm{cm}^{-1}\right)$ & $\mu_{a}\left(\mathrm{~cm}^{-1}\right)$ & $\mu_{s}^{\prime}\left(\mathrm{cm}^{-1}\right)$ & $\mu_{a}\left(\mathrm{~cm}^{-1}\right)$ \\
\hline 0.05 & $9.85 \pm 0.5$ & $0.052 \pm 0.005$ & $11.39 \pm 4.10^{-2}$ & $0.0585 \pm 4.10^{-4}$ \\
\hline 0.1 & $9.8 \pm 0.6$ & $0.102 \pm 0.007$ & $11.7 \pm 5.10^{-2}$ & $0.102 \pm 5.10^{-4}$ \\
\hline 0.15 & $10.35 \pm 0.3$ & $0.150 \pm 0.0035$ & $12.08 \pm 2.10^{-2}$ & $0.142 \pm 2.10^{-4}$ \\
\hline
\end{tabular}

Table 4 


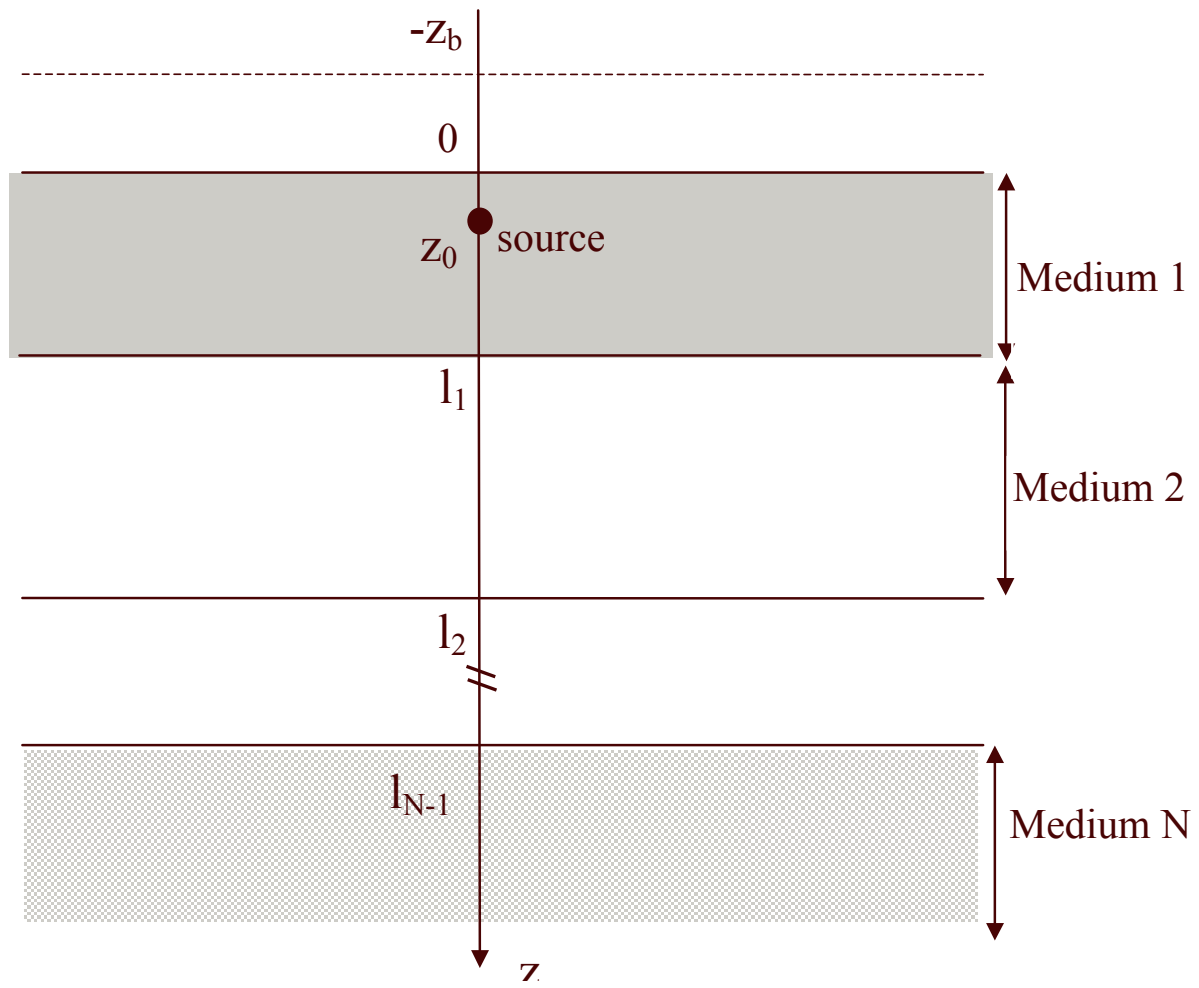

Figure 1 


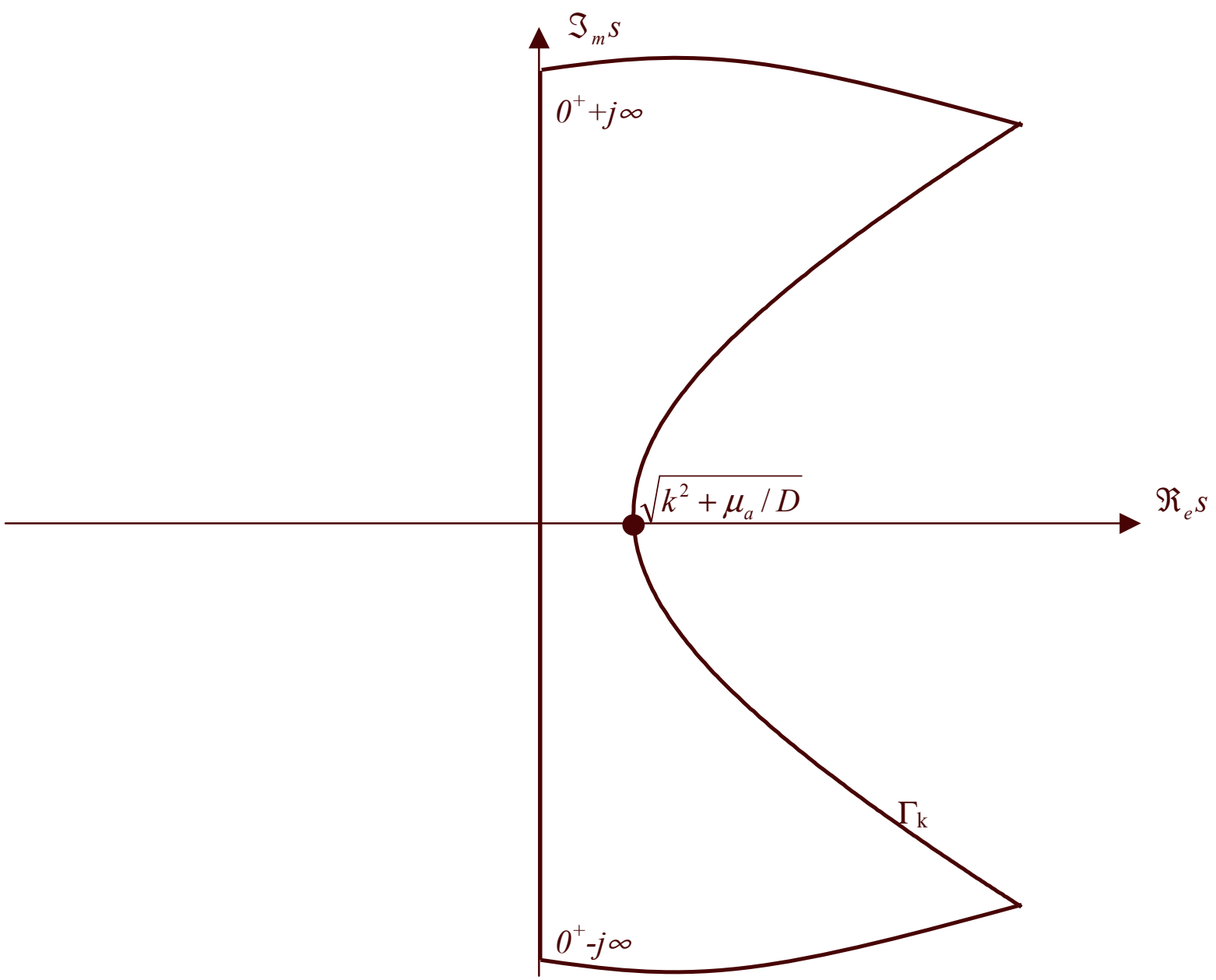

figure 2 
$\ln \mathrm{R}_{\infty}-\ln \mathrm{R}_{\mathrm{n}}$

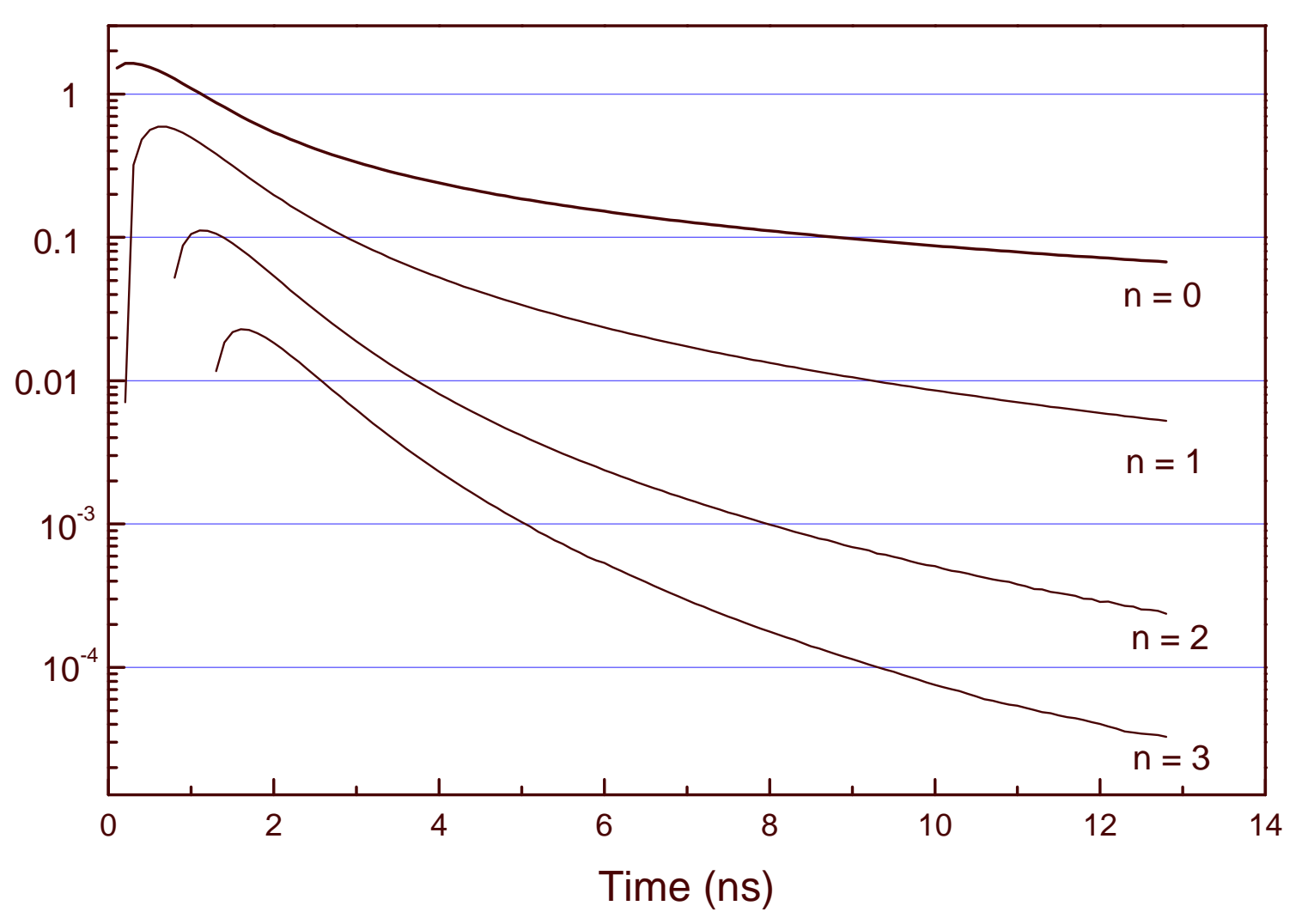

Figure 3 


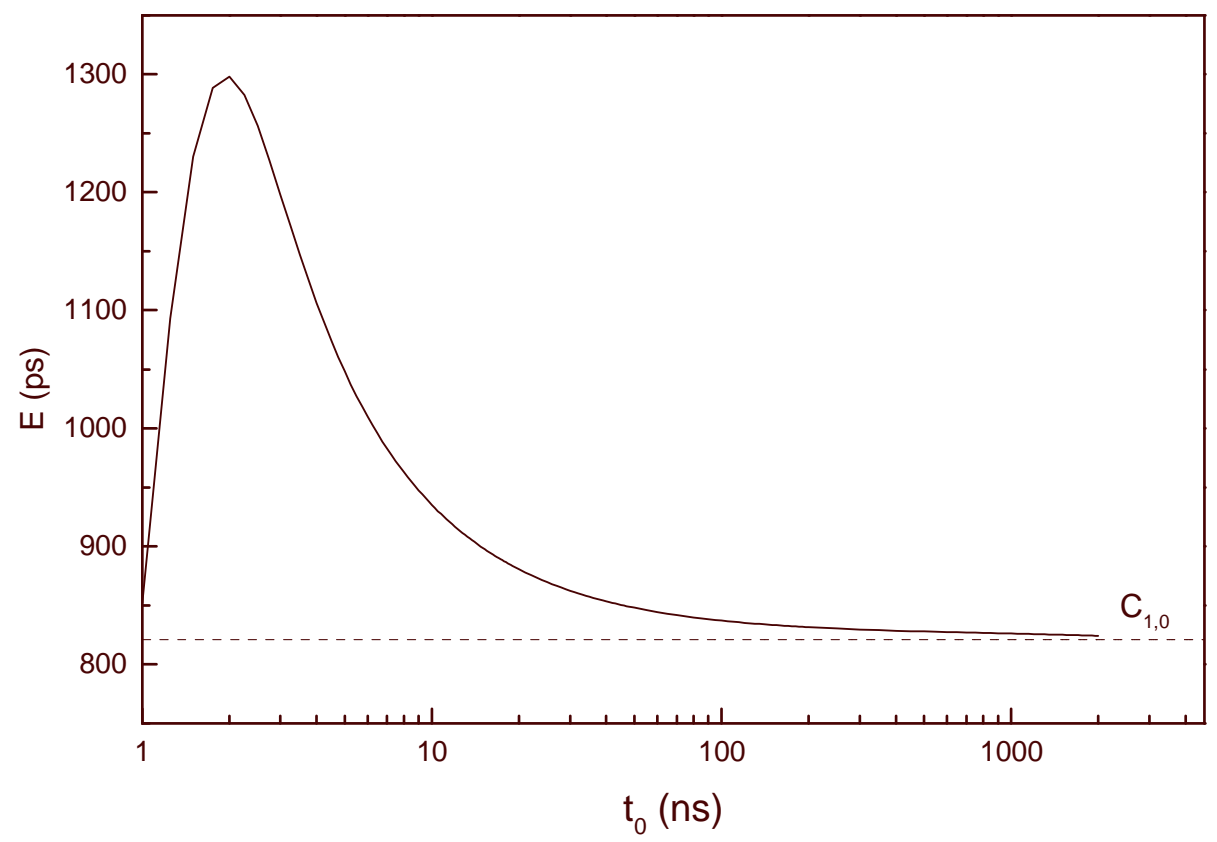

(a)

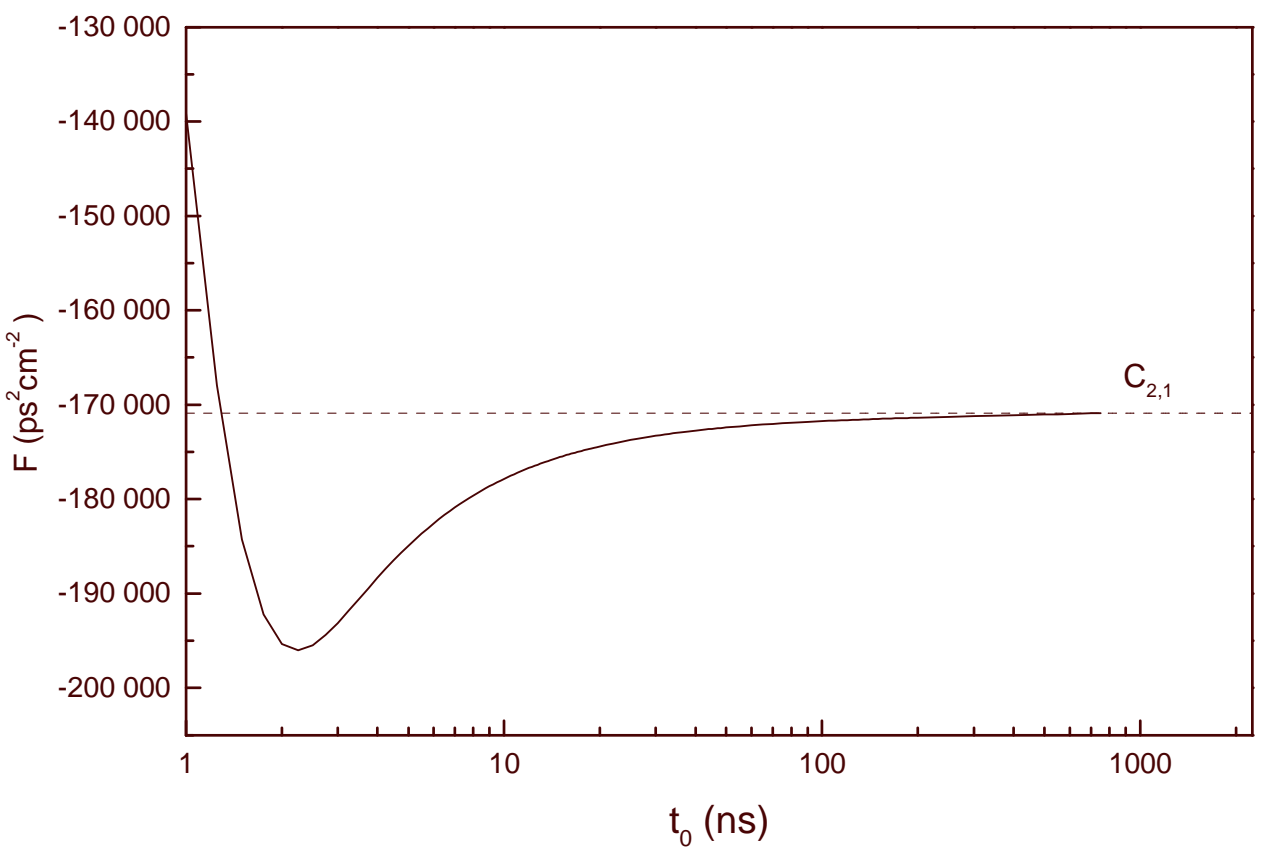

(b)

Figure 4 


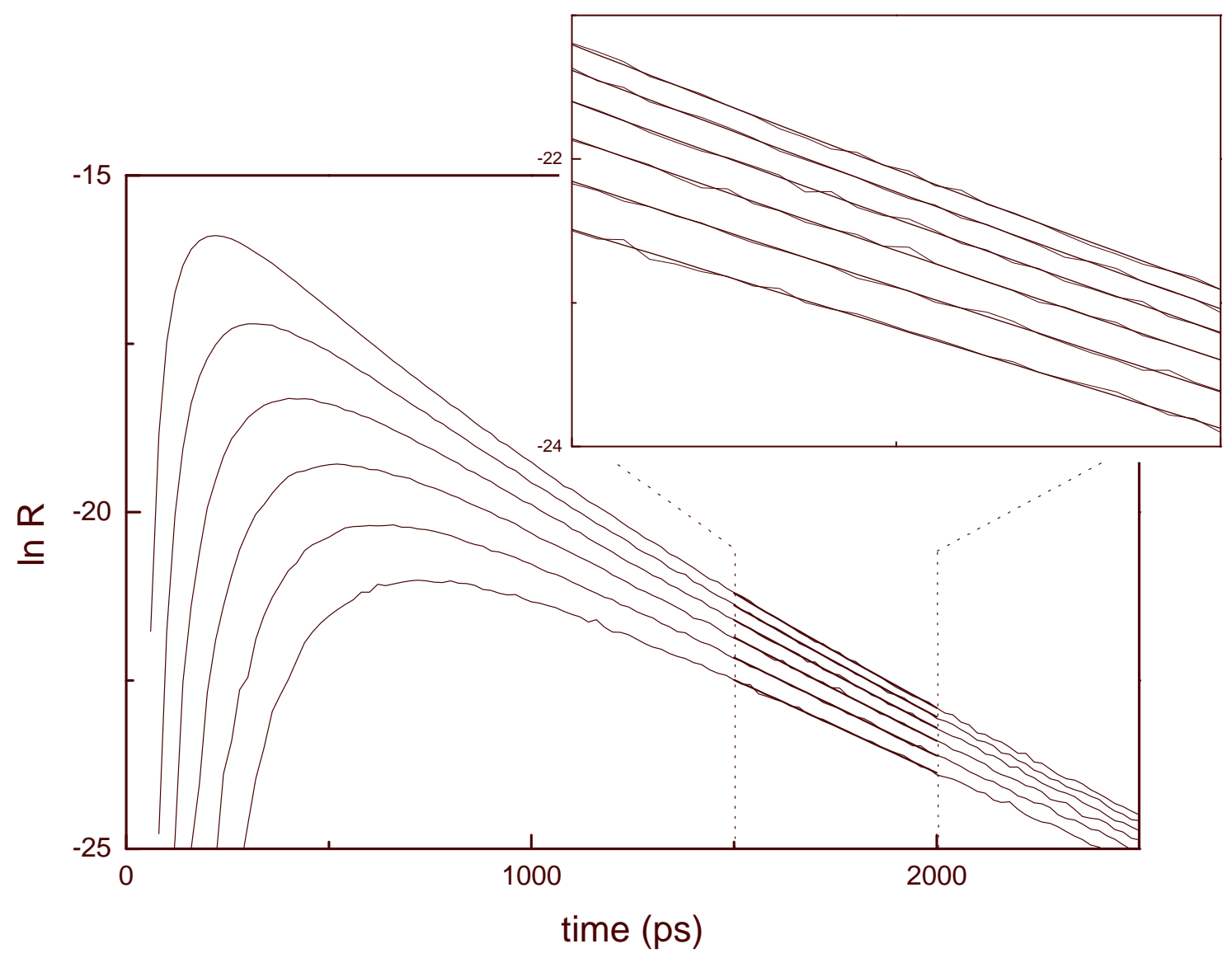

Figure 5 


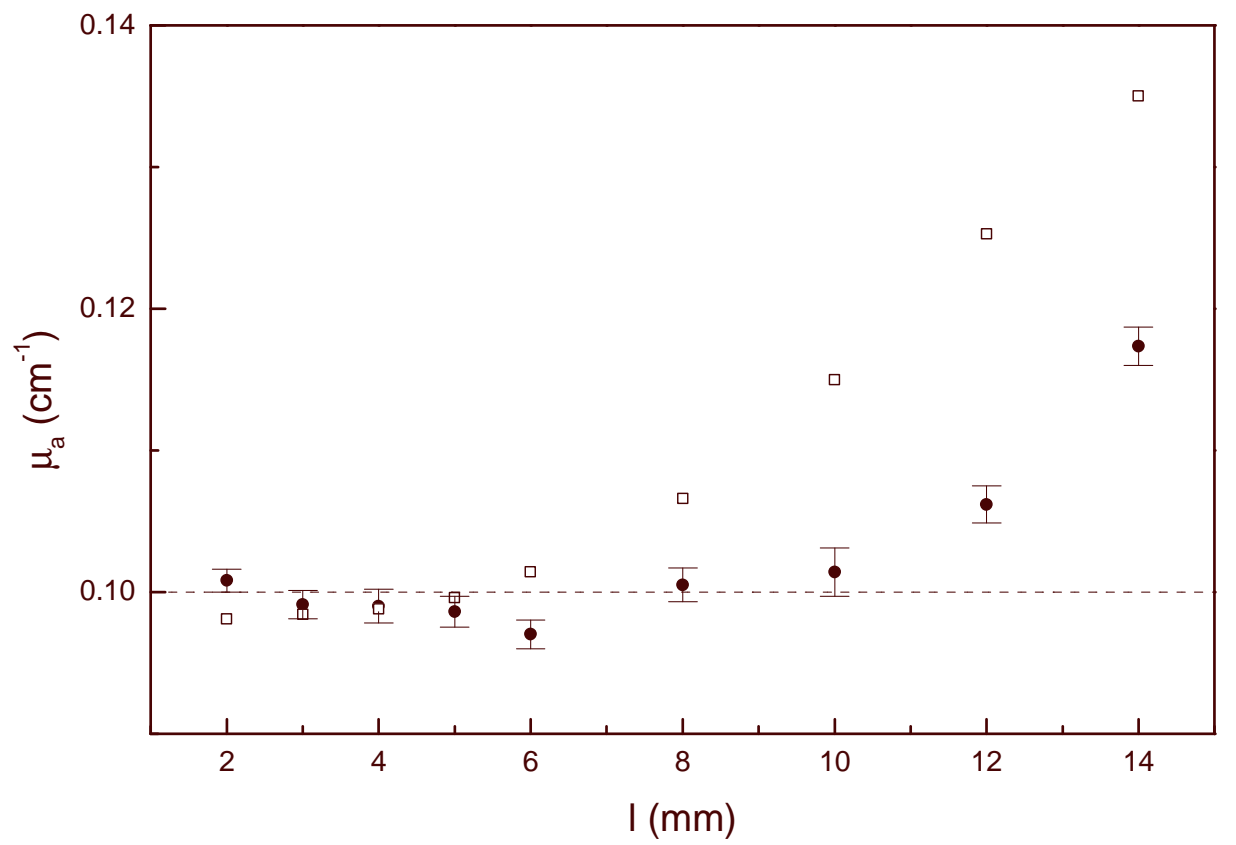

(a)

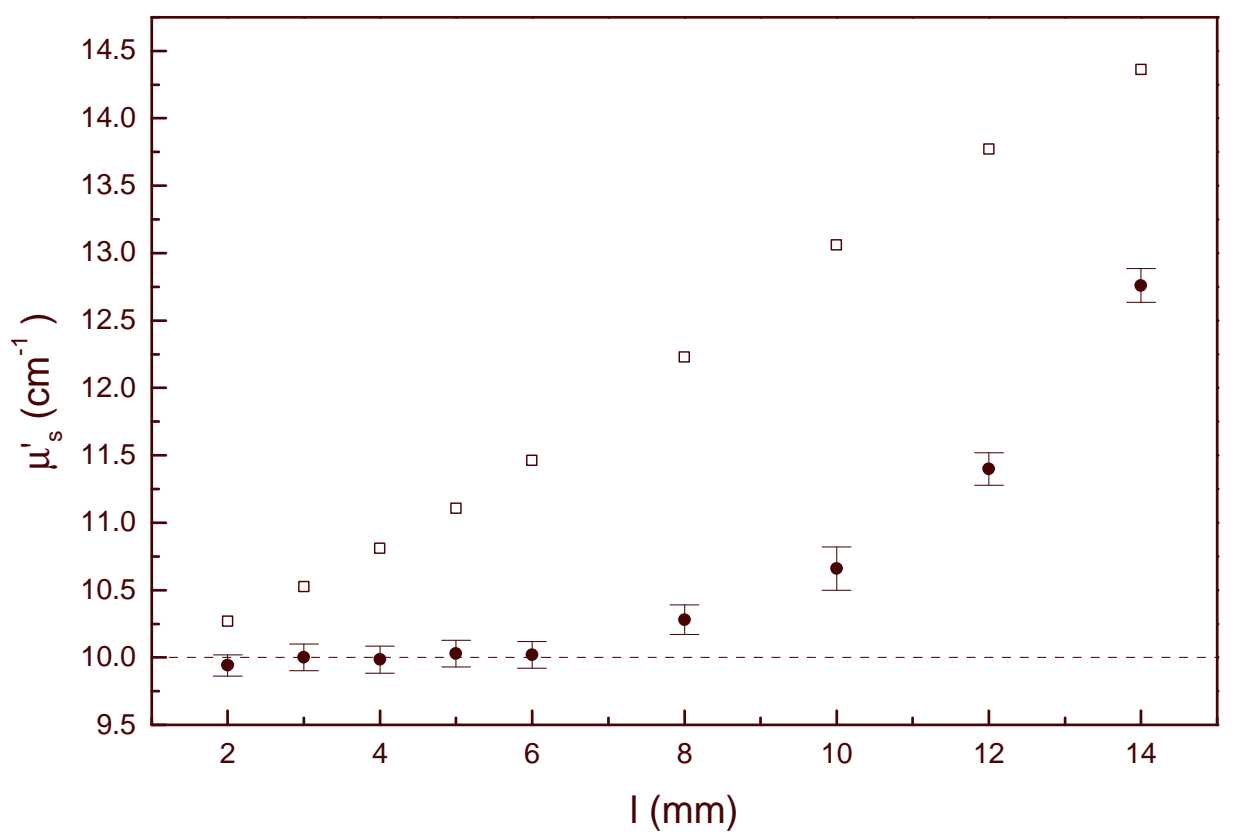

(b)

Figure 6 


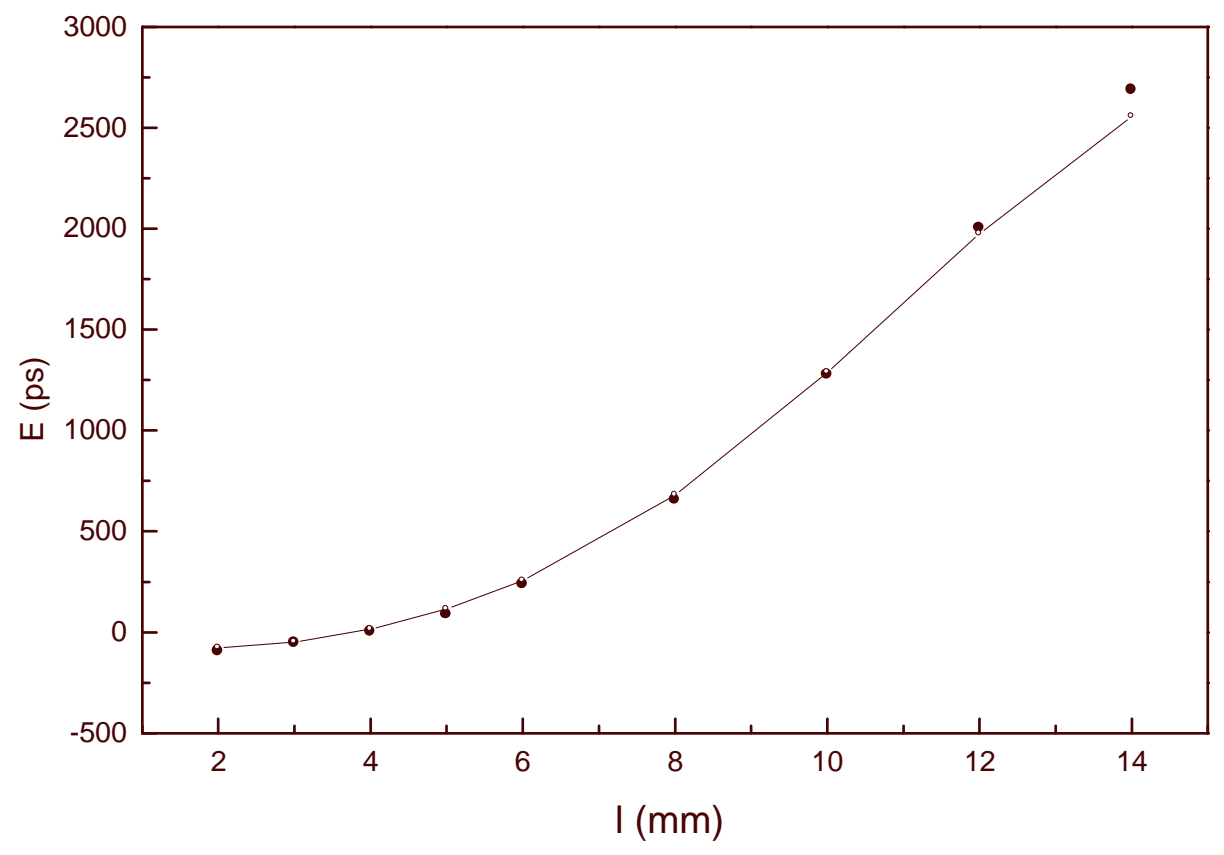

(a)

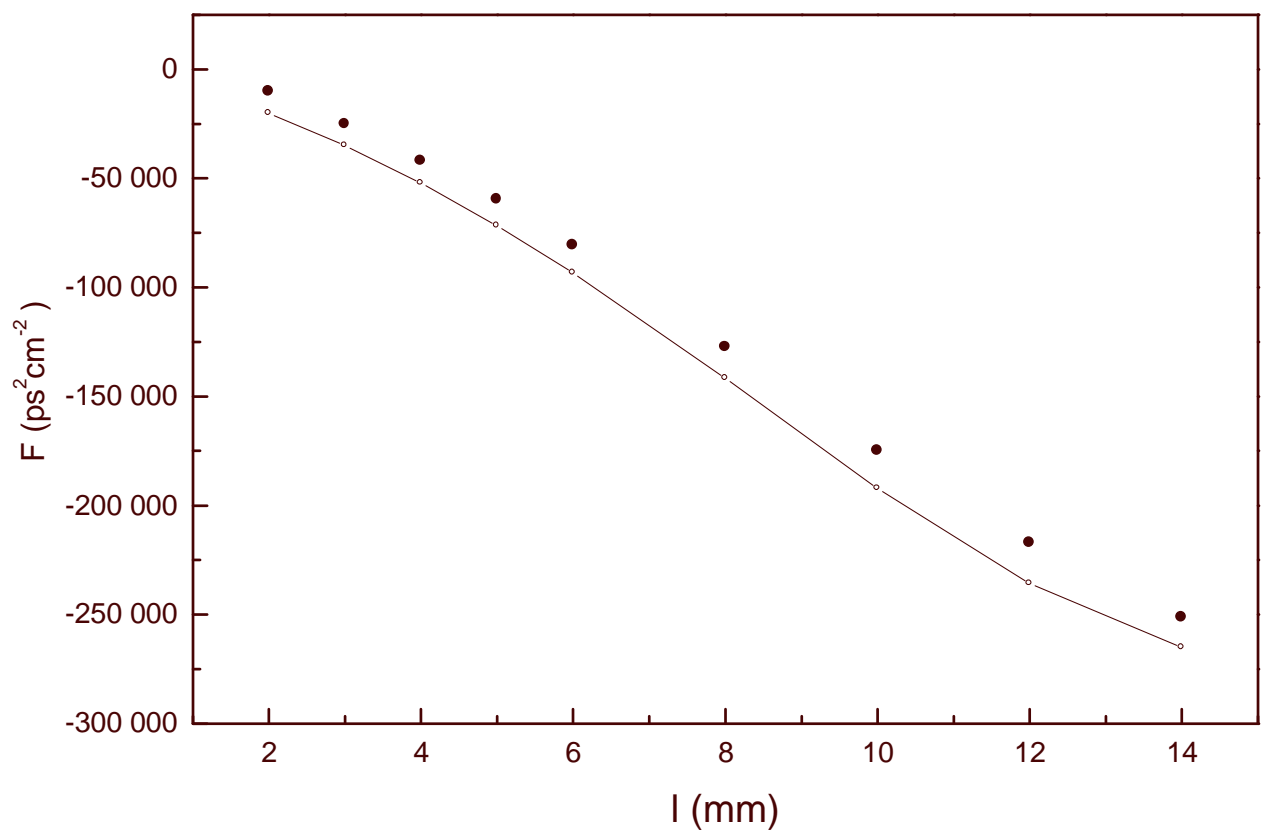

(b)

Figure 7 


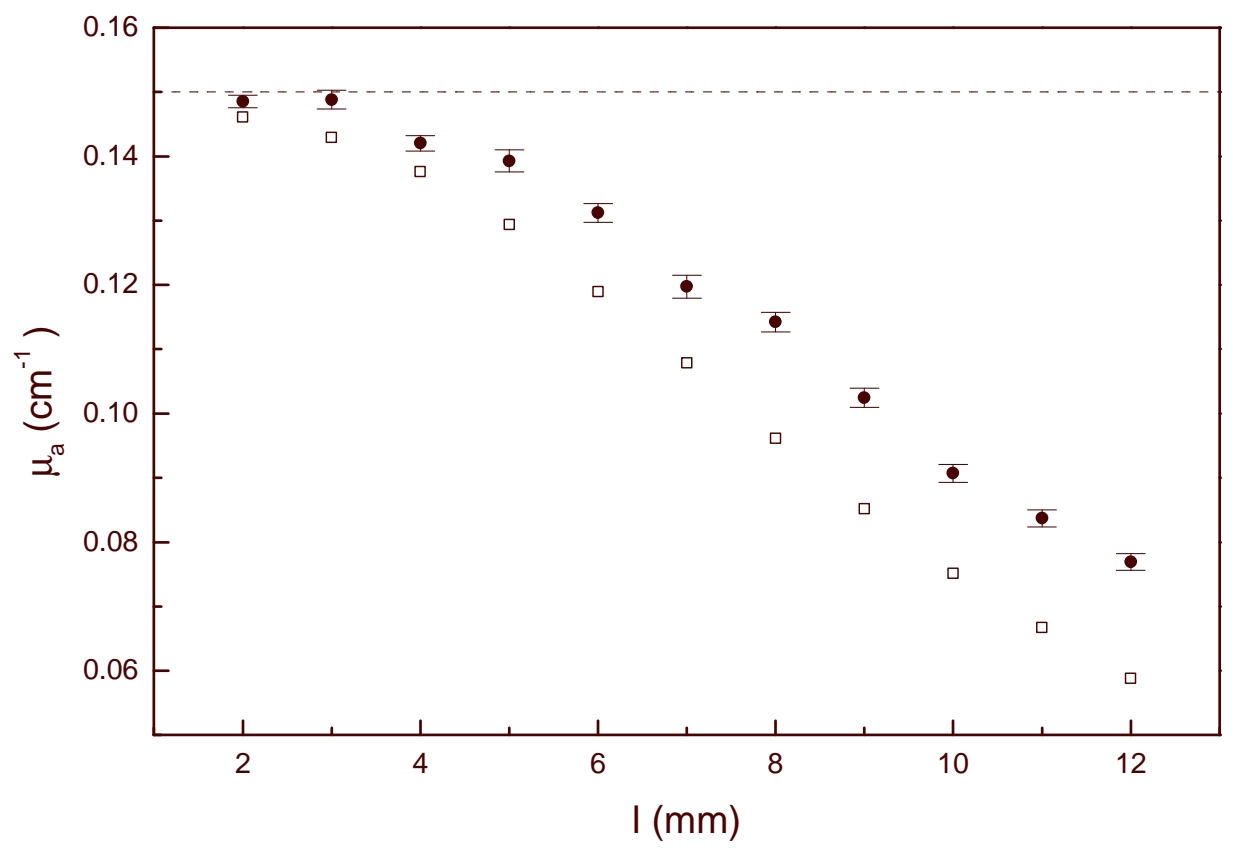

(a)

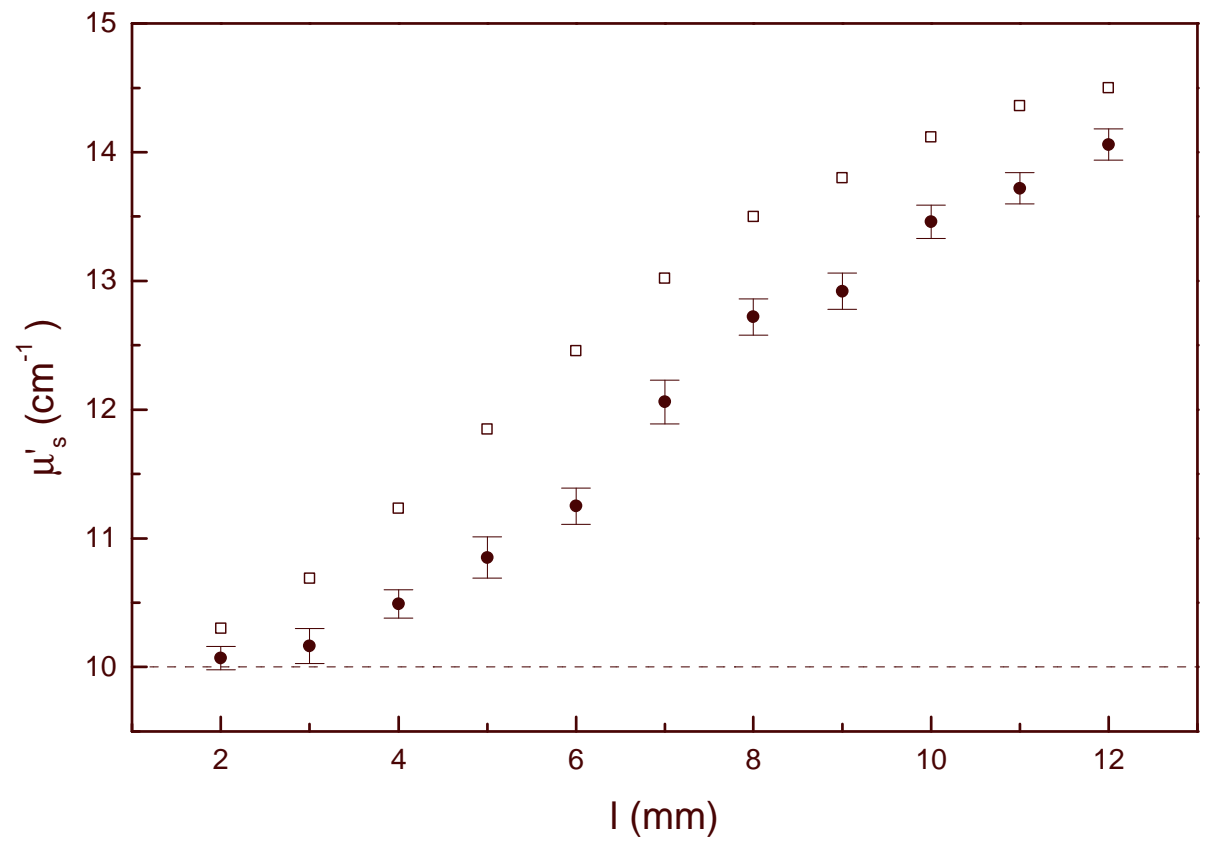

(b)

Figure 8 


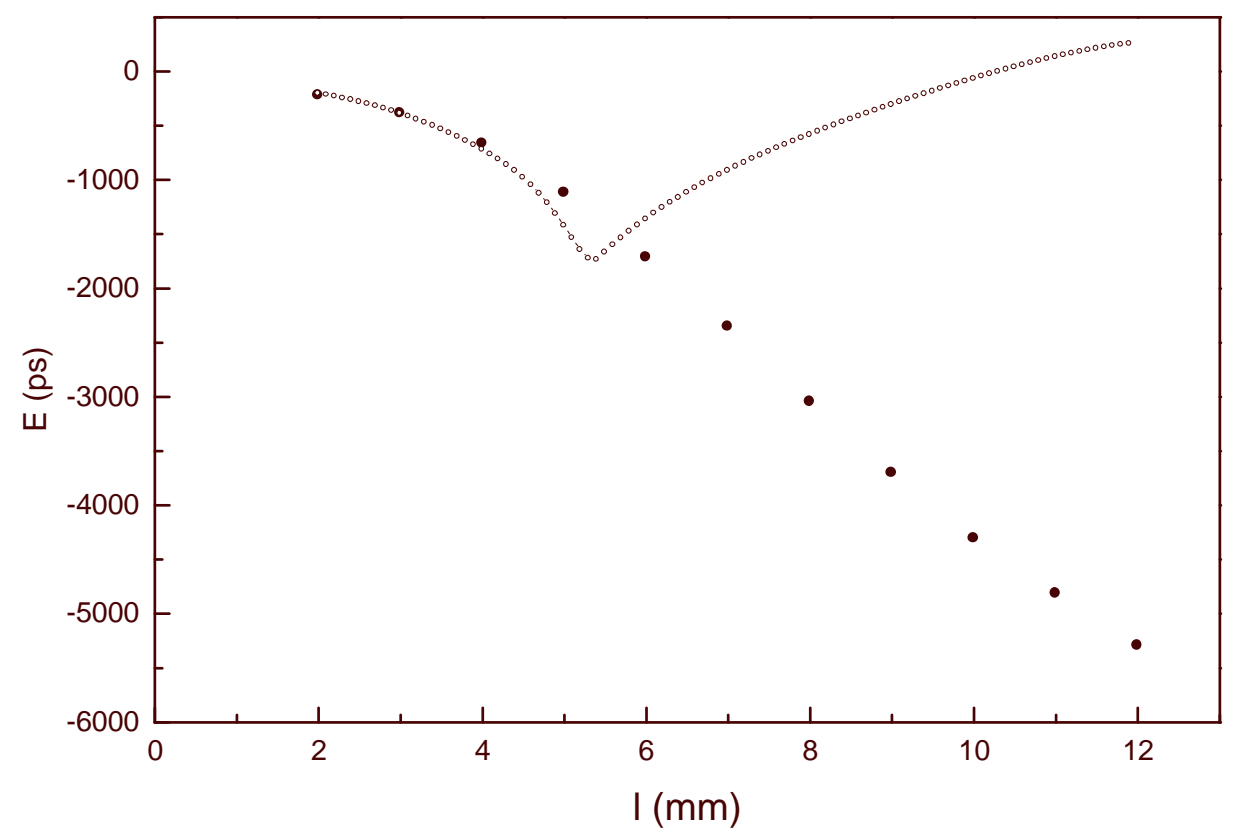

(a)

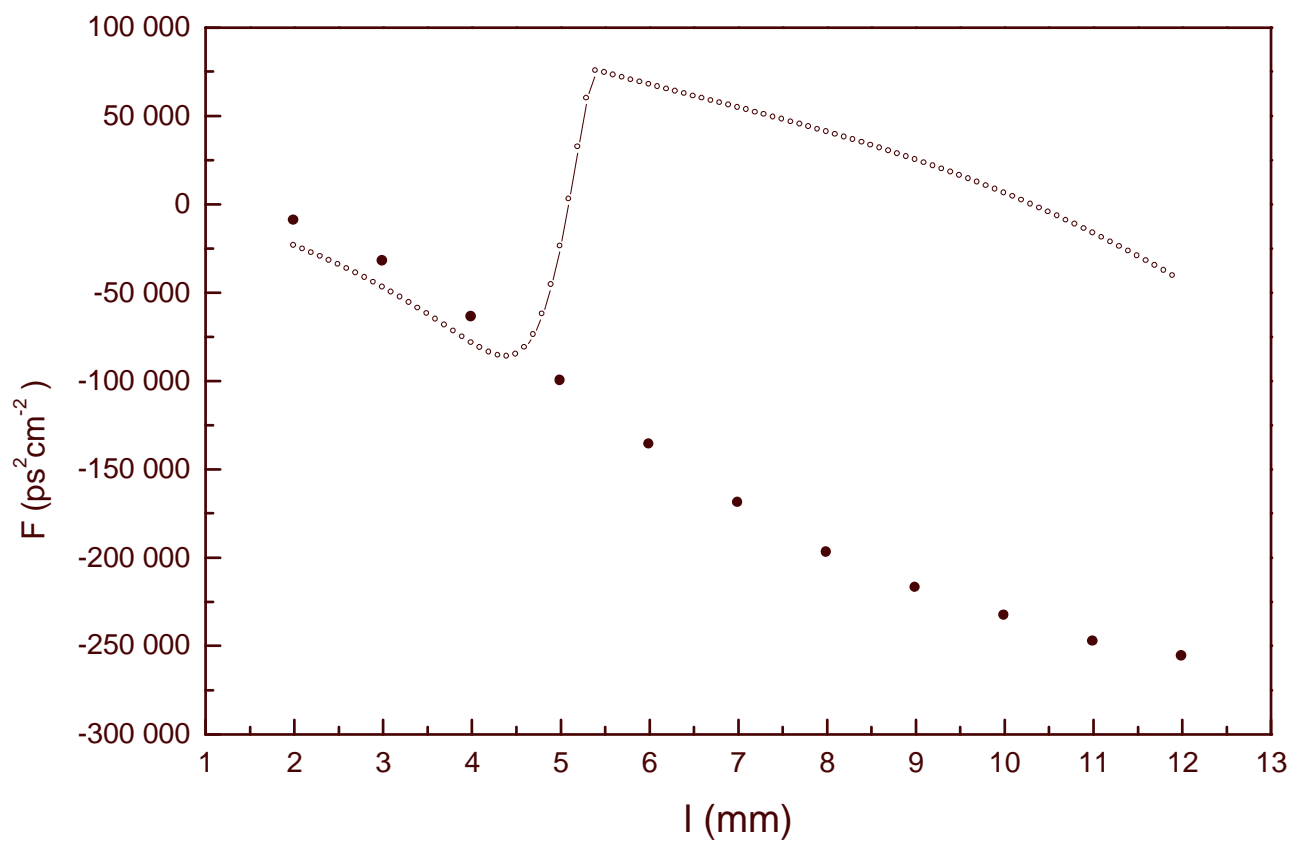

(b)

Figure 9 


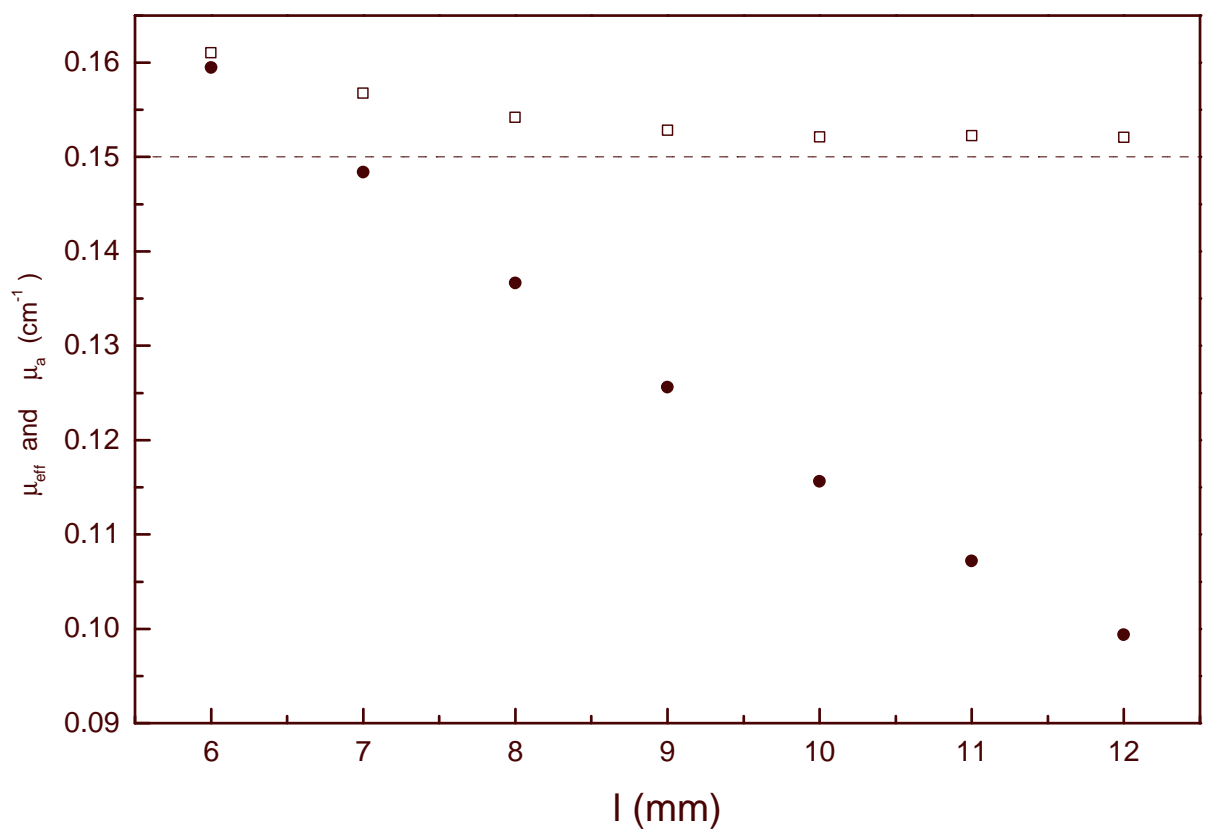

(a)

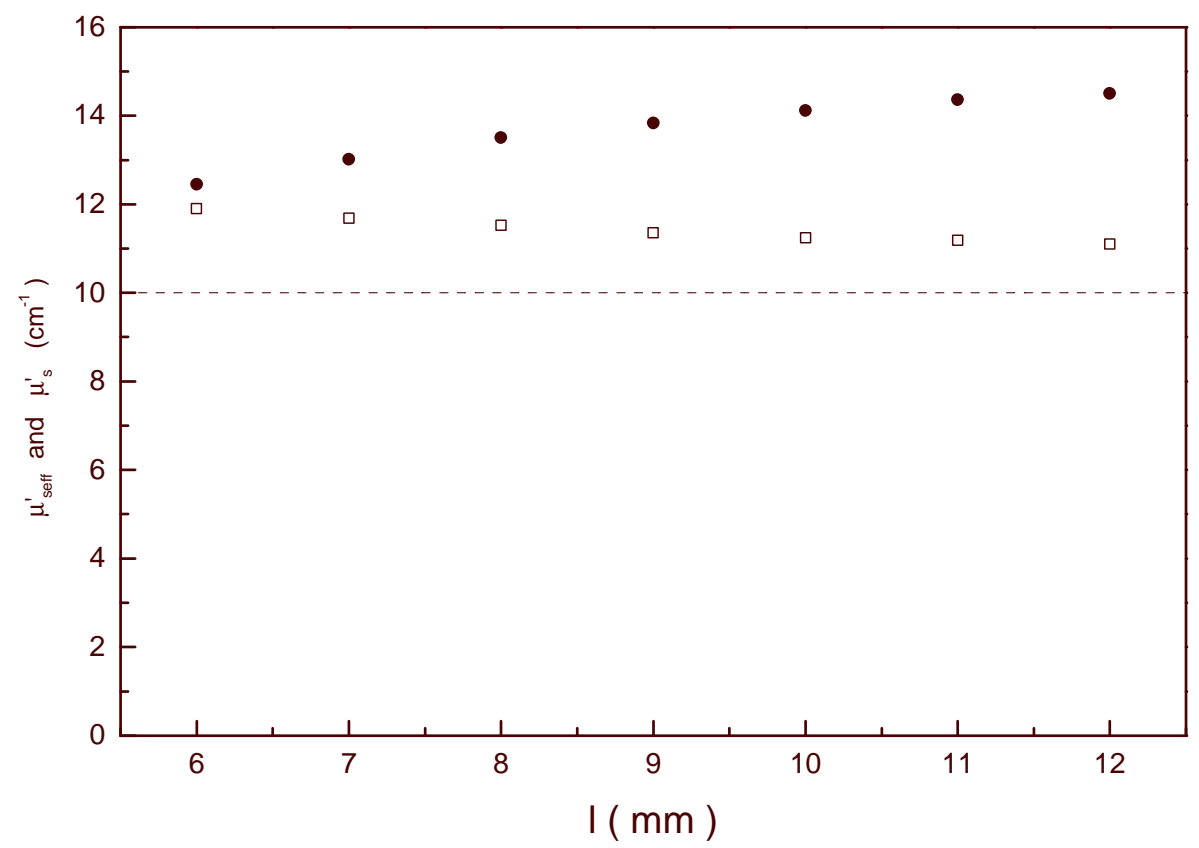

(b)

Figure 10 